\title{
Live visualization of cytosolic and endoplasmic reticulum redox potential in zebrafish embryos reveals region- specific differences
}

Monika Verma ${ }^{1,2}$, Niraj Rajesh Bhatt ${ }^{1,2}$, Aseem Chaphalkar ${ }^{1,2}$, Kriti Verma $^{1}$, Shreyansh Umale ${ }^{1,3}$, Shweta Verma ${ }^{1,2}$, Chetana Sachidanandan ${ }^{1,2,4}$ *, Kausik Chakraborty ${ }^{1,2,4}$ *

${ }^{1}$ CSIR-Institute of Genomics and Integrative Biology, Mathura Road, New Delhi, India 110025

${ }^{2}$ Academy of Scientific and Innovative Research (AcSiR), CSIR-HRDC, Ghaziabad, Uttar Pradesh, India 201002

${ }^{3}$ Indian Institute of Technology Madras, Chennai, Tamil Nadu, India 600036

${ }^{4}$ Equally contributing senior authors

*Correspondence may be addressed to kausik@igib, chetana@igib.in 


\begin{abstract}
Redox homeostasis is an integral part of many cellular processes and its perturbation is associated with conditions such as diabetes, aging, and neurodegenerative disorders. Redox homeostasis or redox potential in organelles is maintained within a particular range to facilitate the organelle specific cellular redox reactions. Previous studies using yeast, cell systems, and nematodes have demonstrated the Endoplasmic Reticulum (ER) has a more oxidizing environment while the cytosol exhibits a reducing redox potential. However, we know very little about redox state of ER in the vertebrate systems. We created transgenic zebrafish (Danio rerio) lines with roGFP sensors targeted to the cytosol and ER for studying physiological redox potential at the systems level. In the process, we characterized the ERtargeting signal sequence in $D$. rerio for the first time. Measurements of the redox state in live embryos found that the ER and cytosol exhibit deviations from the expected range in different regions of the developing embryos. The ER is more reducing than expected in certain tissues, and cytosol has oxidizing regions at par with the ER in other parts of the embryo. This was particularly true for the brain which showed the heterogeneity of redox state in both the ER and the cytosol. These differences became even more evident in a transgenic line expressing the more sensitive roGFPiE variant. Thus, live monitoring of redox potential across the developing zebrafish embryos revealed interesting regions of redox heterogeneity at the organism level.
\end{abstract}

Keywords: redox homeostasis, roGFP, redox potential, signal sequence, zebrafish, reducing ER, oxidizing cytosol, 


\section{Introduction}

The endoplasmic reticulum (ER) is the first stop for the proteins going through the secretory pathway and it acts as a hub for oxidative protein folding in the cell (Ellgaard \& Helenius, 2003). Oxidative protein folding in the ER is mediated by redox buffers and oxidoreductases such as the Protein Disulfide Isomerases (PDIs) and ER Oxidoreductin 1 (ERO1), aided by the oxidizing redox potential in the ER (Ellgaard \& Ruddock, 2005). While the ER is oxidizing, other compartments like the cytosol and the mitochondrial matrix maintain a reducing environment to prevent the resident enzymes from oxidation and to facilitate metabolic reactions (Gutscher et al., 2008; Hanson et al., 2004; Hwang, Sinskey, \& Lodish, 1992; Østergaard, Tachibana, \& Winther, 2004). Differences in the redox potential of the ER and cytosol can also be attributed to differences in ratios of reduced and oxidized glutathione (GSH:GSSG). The cytosol has many fold higher concentrations of GSH:GSSG (10:1 to 100:1) as compared to the ER (3:1 or 1:1) (Hwang et al., 1992; Montero, Tachibana, Rahr Winther, \& Appenzeller-Herzog, 2013; Morgan et al., 2013). However, maintaining redox potential is a complex process and apart from the enzymes it also depends on the redox buffers present in the cell. The generation of live probes for redox sensing such as the roGFP variants have revolutionized the field. These sensors, targeted to various cellular organelles have been used to study intracellular differences in redox potential in a number of model organisms such as Saccharomyces cerevisiae, Caenorhabditis elegans, and mammalian cell lines (Ayer et al., 2013; Birk et al., 2013; Hu, Dong, \& Outten, 2008; Romero-Aristizabal, Marks, Fontana, \& Apfeld, 2014).

The ER is multi-functional; in addition to protein folding, it also serves as a compartment for calcium storage and lipid biosynthesis (Glaumann, Bergstrand, \& Ericsson, 1975; Inui, Saito, \& Fleischer, 1987; Lai, Erickson, Rousseau, Liu, \& Meissner, 1988; Vidugiriene \& Menon, 1993). Within a cell, the ER maintains distinct sub-regions for these functions (Baumann \& Walz, 2001; Sitia \& Meldolesi, 1992). Additionally, the ER in different organs in the body have different demands on it (Zheng \& Staehelin, 2001) e.g. the pancreatic cells are secretory in nature with nearly $1 / 3^{\text {rd }}-1 / 5^{\text {th }}$ of the proteome passing through the $E R$, making protein folding the major activity (Ghaemmaghami et al., 2003). Whereas, the ER in muscles serves more as a calcium storage hub (Burdakov, Petersen, \& Verkhratsky, 2005; Takeshima, 2002) (Coussin, Macrez, Morel, \& Mironneau, 2000). Given this, we speculated that the ER in different tissues would adapt to the differences in demand and would exhibit differences in redox potential.

Studies in the fruit fly larvae (Drosophila melanogaster) and nematodes (C. elegans) have shown differences in cytosolic redox potential across different regions of the animal 
(Albrecht, Barata, Grosshans, Teleman, \& Dick, 2011). Apfeld and colleagues showed that insulin signaling was important for the regulation of redox potential in the cytosol of $C$. elegans (Romero-Aristizabal et al., 2014). Morimoto and group demonstrated that the ER becomes less oxidizing while the cytosol becomes less reducing in aging worms (Kirstein et al., 2015). Most of these studies on redox potential, performed in invertebrate models, have focused on perturbations due to chemical or environmental cues, with the assumption that the ER redox potential is constant across tissue types (Kirstein et al., 2015). However, we know very little about physiological differences in redox states of ER and cytosol across tissues in vertebrates.

We chose zebrafish (Danio rerio) as the vertebrate model to explore the redox potential dynamics because the transparency of the embryos allow us to monitor redox states in the live animal using sensors such as roGFP. The embryonic anatomy and molecular pathways are well conserved from zebrafish to human. The chemical permeability of the embryos and larvae also make it easy to expose them to small molecule perturbation. We generated transgenic zebrafish lines ubiquitously expressing the redox sensor, roGFP targeted to the ER and cytosol. During this process, we also characterized the ER-targeting signal sequence in zebrafish for the first time. Our studies showed that ER and cytosol in most tissues had the expected redox states. However, there were interesting deviations from the norm in the brain and some other tissues. These differences in redox states became even more evident when we used roGFPiE, a more sensitive variant of roGFP. Thus, our organelle-targeted redox sensors allowed us to visualize and quantify the redox states of cytosol and ER in various tissues in a live vertebrate model. These tools we have generated would be valuable in exploring the mechanisms for maintaining different redox states in different tissues and would also allow us to understand the changes in redox states induced under pathophysiological conditions.

\section{Results and Discussion}

\section{Generation and characterization of roGFP transgenic lines revealed correct subcellular localization of the sensor}

To investigate if redox potential of the cytosol and the ER varies across different tissues of zebrafish, we generated zebrafish transgenic lines expressing roGFP2 either in the cytosol or the ER (Fig. 1A). The roGFP2 sensors have disulphide bonds engineered in the proteins such that the protein maintains different conformations in reducing and oxidizing milieu. Both the conformations absorb energy at 405 and $488 \mathrm{~nm}$ of light and emit at $505 \mathrm{~nm}$, however the ratio of absorbance at these two wavelengths shifts according to the redox environment 
in which it is present. Absorbance at 405 is higher when the probe is oxidized (therefore the emission at 505 is higher when excited at $405 \mathrm{~nm}$ ), while absorbance at $488 \mathrm{~nm}$ increases when it is reduced (Hanson et al., 2004). So, the values obtained for emission at $505 \mathrm{~nm}$ when excited at $488 \mathrm{~nm}$ divided by the emission at $505 \mathrm{~nm}$ when excited at $405 \mathrm{~nm}$ (488 $\mathrm{nm} / 405 \mathrm{~nm}$ ) acts as a surrogate for the redox status. Fusing roGFP2 to different disulfide catalysts is known to increase the time resolution of recording changes in redox potential (Bhaskar et al., 2014) (Gutscher et al., 2008). However, expression of these catalysts risks perturbing the cellular redox homeostasis.

roGFPiE is a redox sensor engineered to sense relatively oxidizing compartments like the ER (Lohman \& Remington, 2008), so we made another transgenic line targeting roGFPiE to the ER. We generated zebrafish transgenic lines expressing roGFP2 in the cytosol [Tg(actin:cyroGFP)] (hereafter cyroGFP) and ER Tg(actin:eroGFP) (hereafter eroGFP) and rOGFPiE in the ER Tg(actin:ERroGFPiE) (hereafter ERroGFPiE). The actin promoter ensured ubiquitous expression of GFP in the developing embryos (Fig. S2A). The default localization of the roGFP2 expressed would be in the cytosol. However, to target the sensor to the ER we needed to fuse an ER targeting signal sequence to the N-terminus of roGFP2. No previous study has targeted exogenous proteins to the ER in the zebrafish and thus the ER targeting signal for zebrafish was not known. We chose to identify the sequence from GRP78 or Bip, a canonical ER chaperone whose targeting signal sequence is routinely used to target proteins to the ER in S. cerevisiae and mammalian cells (Kanekura, Ishigaki, Merksamer, Papa, \& Urano, 2013; Merksamer, Trusina, \& Papa, 2008). We aligned the protein sequences of GRP78 and HSP70 from Homo sapiens to that of Hspa5 and Hsp8b (HSP70) of D. rerio to identify the putative ER signal sequence. We selected the first 16 amino acids (aa) that appeared to be specific to the ER-targeted Hspa5 and was not present in its cytosolic counterpart, Hsp8b (Figure 1B). These 16 aa of Hspa5 were fused to the Nterminal of roGFP2 and the first 26 aa of Hspa5 were fused to the N-terminal of ERroGFPiE to assess the minimal sequence needed for targeting.

In the embryos, where cells are packed together, it was difficult to visualize the organelles clearly. Thus, we prepared a primary cell culture from $1 \mathrm{dpf}$ (days post fertilization) zebrafish embryos by dissociating the cells from the embryo and allowing them to attach to a substratum (Figure 1C). The GFP fluorescence in the cyroGFP cells was present throughout the cell reminiscent of localization in the cytosol (Figure 1D and S1A). The GFP fluorescence in the eroGFP showed a tubular pattern distinct from the cyroGFP; we observed a perinuclear distribution of the GFP with nuclear exclusion (Figure 1D and S1A). We stained the cyroGFP, eroGFP and ERroGFPiE cells with various organelle trackers to 
visualize organelles clearly. Visual analysis showed a similar pattern between roGFP and ER-Tracker Red in eroGFP line; the MitoTracker Red and Lyso-Tracker Red had very different staining patterns to roGFP. This was confirmed by quantification. The mean colocalization rate of roGFP with ER-Tracker was $63.48 \%$ compared to $25.42 \%$ with MitoTracker Red (Figure1E). Similar trends were observed for the ERroGFPiE line, indicating ER localization (Figure S1C). Thus, it appears that both 16 aa as well as 26 aa can target proteins to the ER and that the 16 aa ER-targeting signal sequence is sufficient. Quantification of roGFP in the cyroGFP line did not show any colocalization with ER or MitoTracker Red (Figure S1B). We further assessed the ER localization of eroGFP by subcellular fractionation and found significant enrichment of GFP in the ER fraction compared to nuclear fraction (Figure S1D). Due to similar densities of ER and mitochondria, it was difficult to separate these two completely using biochemical methods leading to the detection of roGFP in the ER-contaminated mitochondrial fraction as well. However, the biochemical and imaging data, taken together, indicated that the redox sensitive GFPs were localized correctly in their respective compartments.

\section{The eroGFP line does not exhibit ER stress}

Overexpression of exogenous proteins can perturb and stress the cell. We assessed the eroGFP lines for ER stress marker expression. The expression of GRP78 (Hspa5 in zebrafish), known to be upregulated during ER stress (Kozutsumi, Segal, Normington, Gething, \& Sambrook, 1988), did not change significantly in the eroGFP line compared to WT (Figure 2A and B). Manganese superoxide dismutase (SOD2) protects cells against oxidative stress (Kasahara, Lin, Ho, \& Reddy, 2005; Wang, Branicky, Noë, \& Hekimi, 2018). Our studies showed no significant change in Sod2 expression in the transgenic lines (Figure $2 \mathrm{~A}$ and $\mathrm{C}$ ). An increase in disulfide bond formation in the ER due to overexpression of proteins may also result in increase in the reactive oxygen species (ROS) levels (Harding et al., 2003; Zeeshan, Lee, Kim, \& Chae, 2016), especially since eroGFP harbors engineered disulfide bonds (Hanson et al., 2004). We used the DCFDA assay to detect ROS levels and found it to be comparable between the eroGFP and the control embryos (Figure 2D). Thus, the eroGFP transgenic lines did not show any signs of ER stress in normal conditions.

\section{The roGFP lines respond to external perturbations in cellular homeostasis}

For the roGFP transgenic lines to be useful for monitoring redox potential in the animal, the roGFP proteins must be responsive to changes in the oxidative state. We measured the emission peak of the roGFP when excited at $405 \mathrm{~nm}$ and $488 \mathrm{~nm}$ with live emission scans of $4 \mathrm{dpf}$ larvae on the confocal microscope. We found the expected $505 \mathrm{~nm}$ peak when excited at $405 \mathrm{~nm}$ or $488 \mathrm{~nm}$ in all transgenic lines (Figure S3A-E). The head region in eroGFP 
embryos showed peak at $505 \mathrm{~nm}$ upon excitation at $405 \mathrm{~nm}$ or $488 \mathrm{~nm}$ indicating that roGFP is oxidized in the ER, while cyroGFP embryos showed very minimal 405 signal (505 nm emission with $405 \mathrm{~nm}$ excitation) suggesting a reducing milieu (Figure S3A and C). Similar results were also obtained for the trunk region of the larvae (Figure S3B and D). Thus, roGFP2 targeted to ER and cytosol reported the expected relative redox differences between the two compartments. The transgenic line ERroGFPiE also showed two characteristic peaks where the emission signal at $405 \mathrm{~nm}$ was higher than $488 \mathrm{~nm}$, as previously reported for this variant (Figure S3E). The oxidized form of roGFPiE is predominant in the ER (405 peak in Figure S3E). These peaks were absent in the WT zebrafish embryos (Figure S3F) showing the signals to be specific for the fluorescent reporters.

To assess the response of the roGFP lines to perturbations in cellular redox potential we used dithiothreitol (DTT) and tunicamycin (Tm) treatment. To avoid variability due to drug penetration in the embryo, we prepared a primary culture of cells dissociated from $1 \mathrm{dpf}$ embryos. The cells were treated either with $10 \mathrm{mM}$ DTT (reducing reagent) or $6 \mu \mathrm{g} / \mathrm{ml}$ and $10 \mu \mathrm{g} / \mathrm{ml}$ of Tm (ER stress inducer). To test the efficacy of Tm in inducing ER stress in the primary culture cells we collected protein from the cells after 8 hours (hrs) of treatment and performed western blots for ER stress markers. We found upregulation of both Hspa5 and Hsp90b1 (GRP94) (canonical ER stress markers) in the eroGFP cells at both the concentrations of Tm (Figure 3A). All further perturbation experiments were performed after $8 \mathrm{hrs}$ of treatment with $6 \mu \mathrm{g} / \mathrm{ml}$ of Tm. All DTT treatments were done with $10 \mathrm{mM}$ DTT and imaging was started within 1-2 minutes ( $\mathrm{min}$ ) of addition of the compound.

Two different fluorescence measurements were done for both the eroGFP and cyroGFP probes: fluorescence emission from $505-540 \mathrm{~nm}$ upon excitation at $488 \mathrm{~nm}$ and from 505$540 \mathrm{~nm}$ upon excitation at $405 \mathrm{~nm}$. The fluorescence ratio of these two measurements 488 $\mathrm{nm} / 405 \mathrm{~nm}$ were then obtained after analysis and will be henceforth referred to as Ratio-2. Pixel wise frequency distribution of Ratio-2 in eroGFP and cyroGFP cells showed that they followed a roughly log-normal distribution (Figure 3B). We transformed the ratio in log2 scale and set two boundaries for quantitative demarcation of reduced and oxidized regions. R1 boundary was defined as 1 standard deviation more than the mean eroGFP value while R2 was set at 1 standard deviation less than mean cyroGFP value. Pixels with Ratio-2 less than R1 represented 'oxidized' pixels and those with Ratio-2 greater than R2 represented 'reduced' pixels. Pixels between R1 and R2 represented the intermediate between oxidized and reduced state (Figure $3 \mathrm{~B}$ ). For ease of visualization, in the cellular and embryonic images, the oxidized pixels were false-colored red, the reduced pixels were colored blue and the intermediate pixels green. 
We determined the Ratio-2 in the transgenic cells treated with DTT. Treatment of eroGFP cells with DTT resulted in instant and significant increase in the Ratio-2, indicating a change from oxidized to reduced state (Figure $3 \mathrm{C}$ and $\mathrm{D}$ ). Tm was expected to cause ER stress and induce reducing conditions in the ER (Merksamer et al., 2008. Instead, treatment with Tm reduced the Ratio-2 in eroGFP marginally suggesting a shift to oxidized state (Figure 3C, 3D and S4). This trend towards a reduction in the Ratio-2 when treated with Tm was not consistent across different sets of cells (Figure S4); importantly the ER redox potential did not show a shift towards reducing state upon treatment with Tm. The cyroGFP cells did not show any significant change in the Ratio-2 in response to DTT or Tm; likely because the cytosol is already reduced (Figure 3C, 3D and S4). Taken together, the eroGFP and cyroGFP zebrafish lines were able to report the expected redox potentials and respond to external perturbations.

\section{Redox potential of ER and cytosol show region wise differences}

Obtaining a quantitative redox map of zebrafish embryos was fraught with hurdles. High autofluorescence (scatter) in certain regions of the embryo precluded determination of the correct ratio of GFP fluorescence upon excitation at the two different wavelengths. To solve this problem, we needed to determine the contribution of autofluorescence in the different regions of the embryo. We took fluorescence images and collected emission from $505 \mathrm{~nm}-$ $540 \mathrm{~nm}$ upon excitation at either $405 \mathrm{~nm}$ or $488 \mathrm{~nm}$ using WT zebrafish embryos with no roGFP2 expression. While excitation at $488 \mathrm{~nm}$ had negligible autofluorescence, $405 \mathrm{~nm}$ showed high autofluorescence in certain regions of the embryo. Since each embryo has unique variations in anatomical structures it is impossible to create a universal quantitative map of autofluorescence in these embryos. To obtain the contribution of autofluorescence in the $405 \mathrm{~nm}$ excitation channel in the roGFP transgenic embryos we needed to generate an autofluorescence map for each embryo. Deconvoluting autofluorescence for a fish with a roGFP2 probe would require us to determine the contribution of autofluorescence with a proxy measurement at a wavelength where the authentic roGFP2 fluorescence has negligible contribution. We found that WT embryos when illuminated at $405 \mathrm{~nm}$, showed an autofluorescence emission peak around $515 \mathrm{~nm}$ and exhibited a prominent shoulder between $440 \mathrm{~nm}$ and $480 \mathrm{~nm}$ (Figure 4A, upper panel). This shoulder was absent in the fluorescence peak of purified roGFP2 (Figure 4A, lower panel). Thus, we reasoned that autofluorescent regions in a roGFP embryo would also show significant emission (in the shoulder) between $440 \mathrm{~nm}$ and $480 \mathrm{~nm}$ while excited at $405 \mathrm{~nm}$ and that this can be used to differentiate from true roGFP fluorescence. We therefore took an additional image of each embryo with $405 \mathrm{~nm}$ excitation and measured the emission from $440 \mathrm{~nm}-480 \mathrm{~nm}$ (Figure 
4B). This image allowed us to build a quantitative estimate of the autofluorescent regions of the embryo; these regions were excluded from all further analysis.

Another problem we faced while imaging zebrafish larvae was visual access to the whole embryo under the confocal microscope. Due to the thickness of the tissue, we could capture only one of the lateral halves of the larva. As the tail region of the animal is thinner, we could image nearly the whole depth, but the head and trunk containing the visceral organs could be only partially imaged. All confocal scans were performed with the larvae oriented laterally. The 3-D stacked images could then be rotated and viewed from various angles according to requirement (Figure S5). Optical sections were then taken to look closely beneath the surface of the regions we found important. That particular section used in each image is indicated by the schematic of a plane slicing through the 3-D reconstructed image (Figure 5). Once the images were acquired and analyzed, it was a challenge to identify the precise anatomical structures showing interesting redox potential patterns due to lack of reference points in the dark images. Thus, for the current study we have focused our analysis on four virtual slices (1-4) that cover the broad regions of brain and muscle in the larvae (Figure $5 \mathrm{~B}$, $C$, and $\mathrm{D}$ ). We have observed many interesting regions of redox anomaly outside the brain and muscle which are not discussed here. The full dataset in raw format is hosted on the Dataverse server (https://doi.org/10.7910/DVN/PRCFJM) and we hope that researchers would be able to identify their favorite regions from the dataset for further analysis.

To determine the highest and lowest boundaries of Ratio-2, we measured the Ratio-2 for a solution of purified roGFP2 in either DTT or $\mathrm{N}, \mathrm{N}, \mathrm{N}^{\prime}, \mathrm{N}^{\prime}$-tetramethylazodicarboxamide (diamide) to obtain Ratio-2 for the fully reduced form or the disulfide bonded form, respectively. While comparing the Ratio-2 distribution of the recombinant protein roGFP2 with the Ratio-2 of the eroGFP or cyroGFP embryos we observed an inconsistency. The eroGFP embryos that should ideally be fully oxidized showed a ratio lower than the fully oxidized recombinant protein (Figure S6A). Similarly, cyroGFP, ideally fully reduced, showed a ratio lower than fully reduced roGFP2 protein (Figure S6A). This suggested that the Ratio2 distribution might be skewed to a lower value due to the residual background intensity in the $405 \mathrm{~nm}$ excitation channel. The fluorescence of roGFP2 in the $405 \mathrm{~nm}$ channel for both eroGFP and cyroGFP lines is much lower than in the $488 \mathrm{~nm}$ channel. This makes the denominator (during Ratio-2 calculation) more sensitive to background noise (Figure S6C and D). Importantly, the fold difference between the fully reduced and fully oxidized forms of purified roGFP2 was similar to the fold difference in Ratio-2 between cyroGFP and eroGFP in the embryos (Figure S6B). This suggested that the peaks of eroGFP and cyroGFP from the embryo must correspond to the peaks for oxidized and reduced roGFP, respectively, in the context of embryos with non-zero background fluorescence. Henceforth, instead of using 
the theoretical maxima and minima for Ratio-2 we decided to use the empirically determined peaks as maxima and minima for all calculations.

To be conservative in defining unexpected deviations in redox potential we used the eroGFP as controls for cyroGFP. Since the ER-targeted roGFP is considered to be in a completely oxidized state, we picked the median of the eroGFP Ratio-2 as the lowest boundary (R3) for an oxidized state of cyroGFP. Thus, we defined any pixels with a Ratio-2 less than R3 as oxidized. Pixels with Ratio-2 higher than R4 (median + one standard deviation of eroGFP) was defined as reduced cyroGFP (Figure 6A).

Once we applied all the above discussed corrections in the analysis we uncovered an interesting paradox. We found that the cytosolic redox potential was strongly oxidizing (sometimes as much as the ER) in multiple regions of the embryo (Figure 6B and C). This is supported by the frequency histogram (Figure 6D). Could these observations be spurious, or an artifact of the arbitrary division of a normal distribution? We think not, because of three reasons. One, the number of pixels in the lower range of Ratio-2 for cyroGFP was more than expected from a normal distribution obtained after fitting the curve for the top $75 \%$ of the Ratio-2 distribution (Figure 6D). Two, the oxidizing regions (red pixels) form defined clusters surrounded by intermediately oxidizing regions (green pixels) in the embryo (Figure 6B and C) instead of being randomly distributed irrespective of anatomical regions. We confirmed the clustering by confocal imaging at a higher magnification (Figure 6E). Three, the oxidizing and the intermediately oxidizing regions were consistent between different embryos (Figure $6 \mathrm{~B})$. Thus, cyroGFP could delineate regions of the embryo with oxidizing cytosolic redox potential. Closer examination of the images revealed that the highly oxidizing regions of the embryo were absent around the skeletal muscles and were enriched in areas surrounding the midbrain and forebrain regions (Figure 6B, C, and E). This was true for multiple $3 \mathrm{dpf}$ embryos (Figure 6). Close observation also revealed that the oxidizing regions formed a layer surrounding the 'normal' reducing regions deeper in the brain (Figure 6E). From our data it is not clear whether the oxidizing layer is within the brain tissue or is a layer outside of the brain.

\section{Map of the redox potential of ER using eroGFP}

For analyzing eroGFP and for creating a conservative map of unexpected deviations in redox potential in the ER we used the cyroGFP peaks as controls. We defined the median Ratio-2 of cyroGFP (R6) as the boundary for identifying anomalous reduced pixels in the eroGFP embryos. Pixels with Ratio-2 less than R5 (one standard deviation lower than the median of cyroGFP) were defined as regions with eroGFP in oxidized state. It must be noted here that the boundaries $(R 5, R 6)$ defined for eroGFP are distinct from the boundaries 
defined for cyroGFP (R3, R4) (Figure 7A). This gave us a conservative map of eroGFP redox potential.

As expected, ER in most regions of the embryo was oxidizing in nature (Figure 7B and $\mathrm{C}$ ). However, a transverse section of the 3D reconstructed images showed a layer with ER in a strongly reducing state just below the skin of the embryo. We checked the Ratio-2 distribution of eroGFP embryo and found that indeed there were many pixels in the intermediate and strongly reducing state than expected from a normal distribution shown by fitted black line (Figure 7D). Transverse section slices confirmed that the strongly reducing pixels (blue/green) were present as a layer surrounding the embryo, suggesting a layer of cells on or below the skin (Figure 7C). Small defined regions with pixels in the intermediate reducing state were present in the hindbrain and forebrain (Figure 7B, and $C$ (last two panels)). These results suggested that there are regions in the embryo that harbor ER with lower redox potential than expected. However, roGFP2 is not a very sensitive probe for ER potential measurement because it is oxidized well below $(-272 \mathrm{mV})$ the redox potential of ER $(-180 \mathrm{mV}$ to $-235 \mathrm{mV})$. Thus, we chose the ERroGFPiE line to characterize differences in ER redox potential across different regions of the embryo.

The ERroGFPiE probe indeed accentuates the deviations-from-expected in the redox potential of the ER across the embryo as seen from the frequency distribution of Ratio-2 for ERroGFPiE (defined as Ratio-iE henceforth) (Figure 8A). Distribution of Ratio-iE did not fit well to a single peak or a double peak normal distribution and consists of at least 3 underlying normal distributions consistent among the different embryos tested (Figure 8A). The peak of the distribution of lowest Ratio-iE corresponds well with the ratio obtained from completely oxidized roGFPiE whereas the peak of the highest distribution corresponds well with the completely reduced roGFPiE (Figure 8A). Thus, roGFPiE does not suffer from the drawback of autofluorescence that shifted the ratio of eroGFP embryos towards lower values. This is primarily due to higher fluorescence of ERroGFPiE in the $405 \mathrm{~nm}$ channel. Conservation of the distributions among different embryos also reinforces the observation that there are hyper-reducing regions in the embryos.

We defined the cutoffs for Ratio-iE based on the ratios obtained from measurements of the purified roGFPiE protein. Pixels having Ratio-iE more than the median Ratio-iE of reduced roGFPiE (DTT treated; R8 boundary) were marked as reducing regions (blue pixels), whereas pixels having Ratio-iE lower than that of oxidized roGFP-iE (diamide treated; R7 boundary) marked regions with oxidizing redox potential (red pixels) (Figure 8B). The mild deviations seen in the eroGFP embryos became clearer in ERroGFPiE as patches of reducing regions in the forebrain (blue pixels) (Figure $8 \mathrm{C}$ ). From the transverse optical sections, we could see conserved regions of reducing state at the caudal limits of the forebrain and the surface of the midbrain (Figure $8 \mathrm{D}$ ). We also observed reducing regions 
possibly around the pericardium (Figure $8 \mathrm{E}$ ). Images were taken at higher magnification to confirm the locations of the reducing regions in the ERroGFPiE embryos (Figure S7A and $B)$. At 40X magnification we were able to observe similar striations of reducing regions in the forebrains (Figure S7B). The trunk region also had striations of reducing regions paralleling the chevron-shaped muscles (Fig. S7B).

Our results suggest that the ER may not be as uniformly oxidizing throughout the embryo as previously thought (Figure 8A). The distribution of Ratio-iE in the embryos corroborates well with the heterogeneity seen in the redox potential of the ER. The lowest peak in the distribution coincides with completely reduced roGFPiE. The middle peak corresponds to a redox potential of $\sim-231 \mathrm{mV}$ (Table 1 ) and is consistent between the different embryos. This peak corresponds roughly to the reported redox potential of the ER as reported in some of the studies (Delic, Mattanovich, \& Gasser, 2010). Roughly $18 \%$ of the pixels represent the ER in this state but a higher percentage remains in a more oxidized $(\sim 37 \%)$ or more reduced $(\sim 45 \%)$ state (Table 1$)$. This data along with the images suggests that there is not a single expected redox potential of the ER in the embryo. It is important to note that the pseudocoloring is conservative and isolates only high-confidence hyper-oxidizing and hyperreducing regions. Since the middle peak significantly overlaps with the low and high peaks it is difficult to illustrate the range of heterogeneity using pseudo-colors. The heterogeneity in redox potential may explain the range of redox values of the ER that have been measured in single cell organisms or in cell lines (Avezov et al., 2013; Brach et al., 2009; Schuiki, Zhang, \& Volchuk, 2012; van Lith, Tiwari, Pediani, Milligan, \& Bulleid, 2011). Taken together, our study revealed that the redox state of cytosol and ER across the vertebrate embryos is not uniform and has many regions of heterogeneity.

\section{Limitations of the study}

While we base our conclusions on our findings, there are certain limitations to our study that need to be considered for future work in this area. We have assumed that the ER-targeting signal and the ER-retention signal are equally efficient in different cell types. There is currently no such report in zebrafish or any other vertebrate systems. Since Bip (GRP78/Hspa5) is a canonical ER chaperone in all tissues, we assumed the same to be true for zebrafish. In support of this assumption, we found that Hspa5 showed ER localization patterns in all the different cells we observed in our embryonic primary culture.

\section{Conclusions}

Within the scope of these limitations, in this study we report three major findings. We describe the first ER-targeting signal sequence for zebrafish tissues. We discovered that the 
redox state of the endoplasmic reticulum is not uniform across different regions and tissues of the embryo with a distribution that ranges between hyper-oxidizing and hyper-reducing. We also observed spatial differences in cytosolic redox state in the zebrafish embryo. We postulate that the redox state of ER and cytosol in different tissues are maintained according to the different functional demands on that tissue. Further work in this exciting area will reveal how each cell maintains their homeostatic redox potential.

\section{Author contributions}

K.C., C.S. and M.V. conceptualized and designed the work. K.C. and C.S. supervised the work. M.V. generated cyroGFP and ERroGFPiE transgenic line and performed all the zebrafish experiments. N.R.B. and K.V. generated eroGFP transgenic line. A.C. did biophysics experiments. S.U. helped in the MATLAB script writing and analysis. S.V. helped in the single cell suspension experiment. Manuscript writing was done by K.C., C.S., and M.V. with inputs from all the authors. All authors read and edited the manuscript.

\section{Acknowledgments}

We are grateful to Sir James Remington for providing us the roGFP plasmids which we used for generation of transgenic lines. We thank Dr. Sridhar Sivasubbu (CSIR-IGIB) for providing pSS550 zebrafish expression vector. This work was primarily funded by BSC0124 grant by CSIR to KC along with IGIB core funding. We thank the CIF facility of CSIR-IGIB for confocal microscope. Instrument and consumable support were also obtained from DBT-Wellcome Trust India Alliance through intermediate fellowship to K.C. MV acknowledges CSIR; NRB, SV acknowledge UGC; and AC acknowledges DBT for their fellowship.

\section{Competence}

The authors declare no competing interests.

*The abbreviations used in this study are: ER, endoplasmic reticulum; PDI, protein disulfide isomerase; GSH, glutathione reduced; GSSG, glutathione oxidized; dpf, days post fertilization; ss, signal sequence; a.a., amino acids; DTT, dithiothreitol; Tm, tunicamycin; ROS, reactive oxygen species; diamide, $\mathrm{N}, \mathrm{N}, \mathrm{N}^{\prime}, \mathrm{N}^{\prime}$-tetramethylazodicarboxamide. 


\section{Materials and methods}

\section{Zebrafish maintenance and breeding}

Zebrafish were maintained and bred at standard temperature conditions of $28^{\circ} \mathrm{C}$. All zebrafish experiments were performed according to the guidelines issued by the Institutional Animal Ethics Committee (IAEC) of the CSIR-Institute of Genomics and Integrative Biology, India. AB (WT), Tg(actin:eroGFP2), Tg(actin:cyroGFP2), and Tg(actin:ERroGFPiE) are used in this study.

\section{Cloning of roGFP constructs}

Conventional as well as homologous recombination based cloning was done for generating the constructs to be used for transgenic lines. pSS550. roGFP2 and roGFPiE were amplified from pRSETB-ro2 and pQE30-ro1/iE obtained as a kind gift from Dr. James Remington lab. $\mathrm{Tg}$ (actin:eroGFP2) was made from the construct made by restriction digestion based method, where 3 primers (no. 1 to 3 ) were used to add Hspa5 signal sequence upstream of roGFP2. Primers 4 and 5 were used to amplify the final construct and subcloned into the pSS550 vector using restriction digestion sites for Nhel and Clal. All primers used are listed in table1. For generating $\mathrm{Tg}$ (actin:cyroGFP2), roGFP2 was amplified from pRSETB-ro2 with primers 6 and 7 having $15 \mathrm{bp}$ sequence homology to the vector ends obtained after restriction digestion. For $T g$ (actin:ERroGFPiE), roGFPiE was amplified and $78 \mathrm{bp}$ corresponding to Hspa5 sequence signal of 26 aa were added by sequential PCR using primers 8,9 and 10 and 11 and 12. All primer sequences are provided in supplementary table 1. In case of ER transgenic lines, the reverse primer had KDEL sequence as well which is an ER retrieval signal. Each of these, vector and the insert, were then recombined using In-Fusion $\mathrm{HD}{ }^{\circledR}$ cloning kit, where 15 min reaction at $50^{\circ} \mathrm{C}$ was setup with enzyme mix provided in the kit. For all the 3 clonings, the reaction mix was transformed in lab made $\mathrm{DH} 5 \mathrm{a}$ competent cells and colonies were screened the next day.

\section{in vitro transcription}

Tol2 transposase was in vitro transcribed using pCS2 where it was first linearized by using restriction enzyme Notl. The transcript was made using SP6 RNA polymerase using mMessage mMachine kit from Invitrogen with $1 \mu \mathrm{g}$ of template DNA. The plasmid itself had polyA at the 3' end, so addition of polyA tailing step was not required. Quality of RNA was assessed using agarose gel electrophoresis and stored at $80^{\circ} \mathrm{C}$ until further use.

\section{Injection and generation of transgenic lines}

One-cell stage embryos were collected after 15-20 min of removing the dividers. The respective roGFP construct and tol2 transposase mRNA was reconstituted in such a way that $1 \mathrm{nl}$ contained $12.5 \mathrm{pg}$ of each, and hence $1 \mathrm{nl}$ of the volume was injected in one-cell stage embryos of WT background. Water was changed in the evening and GFP positive embryos were screened at 2 or 3 dpf stage using ZEISS AxioScope A1 microscope (with 
Axiocam HRc®). At least 100 GFP positive embryos were put for growing into adults, for each line. After 3 months, progeny from three independent founder lines (obtained by backcrossing with the WT line) were put for growing. Embryos obtained from at least F2 stage or more were used for experiments. Before each experiment, $1 \mathrm{dpf}$ embryos were screened for GFP fluorescence using ZEISS AxioScope A1 microscope and positive embryos were processed.

\section{Zebrafish single cell suspension and primary culture}

GFP positive (obtained from the transgenic lines) and WT embryos were first dechorionated manually and transferred to $1.5 \mu \mathrm{l}$ microcentrifuge tubes (MCTs). 50 and 100 embryos were taken for imaging and western experiments respectively. After dechorionation, deyolking was done by adding $100 \mu \mathrm{l}$ of lab made ringer's solution (constitution as per zfin) for 5 min with intermittent pipetting with $200 \mu \mathrm{l}$ tip. $1 \mathrm{ml}$ of $1 \mathrm{X}$ trypsin-EDTA solution (GIBCO), pre warmed at $29^{\circ} \mathrm{C}$, was added and kept at $29^{\circ} \mathrm{C}$ dry bath. $27 \mu \mathrm{l}$ of $100 \mathrm{mg} / \mathrm{ml}$ collagenase type IV (GIBCO) was also added to it. Pipetting up and down was done using $1 \mathrm{ml}$ tip for disruption of embryos into cells after every $5 \mathrm{~min}$ for a total incubation time of $20 \mathrm{~min}$. Reaction was stopped using $200 \mu \mathrm{FBS}$, kept for 2 min and the suspension was spun at 400 $\mathrm{g}$ for 5 min. Cells were suspended in L15 media with 10\% FBS after 2 washes with chilled 1X PBS. Cell suspension was seeded either in chambered slides or 6-well cell culture plates (precoated with $0.2 \%$ gelatin) for imaging or western respectively. Media was changed the next day and culture was processed for further experiment.

\section{Localization using organelle trackers}

First of all media was removed and $10 \mu \mathrm{g} / \mathrm{ml}$ of Hoechst solution, made in 1X HBSS (Hank's Balanced Saline Solution; GIBCO), was added to the primary culture in chambered slides. After 15 min, organelle trackers, working solutions made in $1 \mathrm{X}$ HBSS were added for 30 min. $10 \mu \mathrm{M}$ ER-tracker, $1 \mu \mathrm{M}$ of each Mito and LysoTracker was used. Two washes were given with $1 \mathrm{X}$ HBSS and the L15 media was added again. Imaging was done using 63X oil objective, 4 zoom in Leica SP8 confocal microscope.

\section{Protein extraction and western blotting}

Embryos were harvested by removing water from the petri plates and transferred to $1.5 \mathrm{ml}$ MCTs. Approximately 39 embryos were taken for western experiments. $120 \mu \mathrm{l}$ of NP40 buffer, with PICs (Protease and Phosphatase inhibitor cocktails), was added to the embryos followed by homogenization using tissue homogenizer (name and company). Spin was given at $16,000 \mathrm{~g}$ for $15 \mathrm{~min}$ and supernatant was collected. For protein extraction from primary culture cells, cells were scraped off in PBS and collected in $1.5 \mathrm{ml} \mathrm{MCTs.} \mathrm{NP40} \mathrm{buffer} \mathrm{with}$ PICs was added and the cell suspension was vortexed for 30 min at $4^{\circ} \mathrm{C}$. Spin was given at $16,000 \mathrm{~g}$ for $15 \mathrm{~min}$ and supernatant was taken. Protein concentration was estimated using BCA kit (name and company). Samples were prepared for SDS page in laemelli buffer with 
$30 \mu \mathrm{g}$ of protein per sample per gel. 10-15\% gels were made using premix Acrylamide:Bisacrylamide (Liqui-gel, 29:1) solution according to the need. Wet transfer of proteins was done on $0.2 \mu$ nitrocellulose membrane (Biorad) at $70 \mathrm{~V}$ for $3 \mathrm{hrs}$. Probing was done with $\alpha$-GFP (1:10,000; rabbit, Abcam), $\alpha-G R P 78$ (1:2000;rabbit, Protein tech), $\alpha-$ GRP94 (1:1,000; goat, enzo), a-Actin (1:2000; mouse, Sigma), a-CoxIV (1:1000, rabbit, Cell Signaling Technology), and $\alpha-S O D 2$ (1:1000, rabbit, abcam). Blots were developed using Crescendo solutions (name and company) in Syngene gel imager (name and company).

\section{ER isolation}

Approximately 200 embryos were harvested for ER isolation and the protocol suggested in Nature protocols, 2013 (Prudent et al 2013; PMID not available) was followed. In brief, embryos were homogenized by tissue homogenizer in $200 \mu \mathrm{l}$ of MB buffer. $800 \mu \mathrm{l}$ of MB buffer was added and the mixture was spun at $1,000 \mathrm{~g}$ for $10 \mathrm{~min}$ to remove debris. The supernatant thus obtained was spun again to get nuclear fraction. This supernatant was spun again at $10,000 \mathrm{~g}$ for $10 \mathrm{~min}$ to obtain mitochondrial fraction as pellet. Spin was given again at $10,000 \mathrm{~g}$ for $10 \mathrm{~min}$ and the final supernatant was subjected to $100,000 \mathrm{~g}$ for $1 \mathrm{hr}$. The pellet thus obtained was considered as ER fraction and supernatant as ER supernatant. The fractions were quantified for protein concentrations and samples were made for SDS PAGE in laemelli buffer with $30 \mu \mathrm{g}$ of protein per gel.

\section{Bacterial expression and purification of roGFP2 and roGFPiE}

Genes for roGFP2 and roGFPiE cloned in bacterial expression plasmids pRSETB-roGFP2 $\left(6 x \mathrm{HIS}, A m p^{+}\right)$and pQE30-roGFP1-iE $\left(6 x \mathrm{HIS}, A m p^{+}\right)$respectively. The plasmids were transformed into E. coli BL21 (DE3) and single colonies were inoculated. The primary inoculums were used to inoculate $1 \mathrm{~L}$ LB with Ampicillin $(100 \mu \mathrm{g} / \mathrm{ml})$ bulk cultures. Protein expression was induced with $0.7 \mathrm{mM}$ IPTG upon reaching OD of 0.6. After induction, cultures were incubated for $18 \mathrm{hrs}$ at $30^{\circ} \mathrm{C}, 200 \mathrm{rpm}$ shaking speed. The cells were harvested and resuspended in a pre-chilled base buffer (1X PBS $+5 \%$ glycerol) containing 2 $\mathrm{mM}$ PMSF. Cell lysis was done using sonication and the lysates were centrifuged at 17,000 $g$ for $1 \mathrm{~h}$ at $4^{\circ} \mathrm{C}$. The supernatants thus obtained were loaded onto separate gravity-assisted Ni-NTA agarose affinity chromatography columns pre-equilibrated with chilled base buffer. The columns were washed with 10 column volumes of base buffer containing $5 \mathrm{mM}$ imidazole. Proteins were eluted with 2 column volumes of base buffer containing $500 \mathrm{mM}$ imidazole. The imidazole was removed by buffer exchange with base buffer at $4^{\circ} \mathrm{C}$ in Amicon Ultra-15 filter unit NMWL 3kDa (Millipore).

\section{Redox titration of roGFP2 and roGFP1-iE}

Based on previous protocols, roGFP2 and roGFPiE were titrated in GFP redox buffer (75 $\mathrm{mM}$ HEPES + $125 \mathrm{mM} \mathrm{KCl}+1 \mathrm{mM}$ EDTA, pH 7.4) containing 0-10 mM DTT (DLdithiothreitol; as a reducing agent) and $10-0 \mathrm{mM}$ diamide (as an oxidizing agent) in a 
reciprocal manner with steps of $1 \mathrm{mM}$ initially for standardizations. Then, 10 concentrations of reducing:oxidizing agents were taken between 4 to $6 \mathrm{mM}$. The titration was set up in a black opaque 96 well plate (compatible with fluorescence measurement). Final volume of redox reaction was kept at $100 \mu \mathrm{l}$ and final concentration of GFP at $10 \mu \mathrm{M}$. The mixture was incubated in dark for $1 \mathrm{hr}$ at room temperature. Finally, the fluorescence intensity of respective wells was measured in TECAN Infinite M200 Pro ELISA plate reader (operated by TECAN I Control V3.3.10.0 software). The excitation wavelength was set at $405 \mathrm{~nm}$ and $480 \mathrm{~nm}$ with emission wavelength kept constant at $520 \mathrm{~nm}$. The data thus generated were processed and plotted in Microsoft Excel.

\section{DCFDA assay for ROS estimation}

ROS estimation was done in 96 well plate using the cell-permeant 2',7'dichlorodihydrofluorescein diacetate (H2DCFDA) (thermo) dye and each well had 3 embryos. 12 such wells were used for one replicate of each line, so one replicate represents data from 3 embryos. Embryos in well with $10 \mathrm{mM} \mathrm{H}_{2} \mathrm{O}_{2}$ was used as a positive control. 200 $\mu \mathrm{l}$ of water was added to each well and a before reading was taken. Then excess water was removed and $200 \mu \mathrm{l}$ of $2.5 \mathrm{mM}$ DCFDA was added in each well, incubated at $37^{\circ} \mathrm{C}$ for $2 \mathrm{hrs}$ and readings were taken in TECAN Infinite M200 Pro ELISA plate reader at $488 \mathrm{~nm}$.

\section{Drug treatments}

Tunicamycin (Sigma) was used at 6 and $10 \mu \mathrm{g} / \mathrm{ml}$ for $8 \mathrm{hrs}$ in primary cells according to the need for imaging as well as western experiments. $0.5 \mathrm{mM}$ DTT (Sigma) treatment for $8 \mathrm{hrs}$ was given to embryos for western while $10 \mathrm{mM}$ DTT was given and cells were immediately imaged for redox readings within $1 \mathrm{~min}$.

\section{Redox imaging}

Embryos were molded first in low melting agarose and then redox imaging was done using leica SP8 confocal microscope with 10X objective; while $63 \mathrm{X}$ objective, zoom 4 was used for cells. First low melting agarose (invitrogen) was heated to boil and kept at $36^{\circ} \mathrm{C}$ in a dry bath for equilibration. Embryos were anaesthetized using $0.004 \%$ Tricaine (Sigma) for 1 min or till it stopped moving, put in agarose for equilibration for a few seconds and immediately put as drop on rectangular coverslip. Embryos were molded in lateral position or according to the need using ZEISS light microscope.

Sequential scan setting for imaging was used in a confocal microscope for redox imaging. In brief, there were four settings at which embryos were imaged called channels. For embryos, channel 0- excitation (ex): $488 \mathrm{~nm}$, emission (em): $505-540 \mathrm{~nm}$, channel 1: bright field, channel 2: ex: $405 \mathrm{~nm}$, em: 440-480 nm, and channel 3: ex: $405 \mathrm{~nm}$, em: 505-540 nm. For cells the same settings were used except emission collected for excitation at $488 \mathrm{~nm}$ and $405 \mathrm{~nm}$ in channel 0 and 3 was from $505-530 \mathrm{~nm}$. The obtained data was processed for 
image analysis. Channels were changed between frames for imaging. Here channel 2 readings were procured for subtraction of background fluorescence.

\section{Image analysis}

Images were extracted from .lif (format of leica microscope) files in.tiff format as grey scale images. These were processed in MATLAB using in-house generated script. The pseudo code of MATLBA script is as shown in steps below:

1. Three frames of images read (image $1=505 / 488$, image $2=505 / 405$, image $3=450 / 405$ (emission $\mathrm{nm}$ /excitation $\mathrm{nm}$ )).

2. The intensity of a pixel is calculated as an average of surrounding pixels ( 1 pixel width) in the $x$ y plane for image 1 and image2.

3. All pixels where the fluorescence in image $1<$ CUTOFF, is replaced by zero in image 1 and image2.

4. All pixels where the fluorescence in image $3>$ BACKGROUND_CUTOFF is replaced by zero in image1 and image2.

5. The ratio of image $1 /$ image 2 is saved as the ratio file containing the ratio information for each of the pixels.

6. The pixels are colored according to the criteria set in the different conditions. The Ratio-2 images thus obtained were viewed in 3D viewer in ImageJ.

\section{References}

Albrecht, S. C., Barata, A. G., Grosshans, J., Teleman, A. A., \& Dick, T. P. (2011). In vivo mapping of hydrogen peroxide and oxidized glutathione reveals chemical and regional specificity of redox homeostasis. Cell Metab, 14(6), 819-829. doi: 10.1016/j.cmet.2011.10.010

Avezov, E., Cross, B. C., Kaminski Schierle, G. S., Winters, M., Harding, H. P., Melo, E. P., . . Ron, D. (2013). Lifetime imaging of a fluorescent protein sensor reveals surprising stability of ER thiol redox. J Cell Biol, 201(2), 337-349. doi: 10.1083/jcb.201211155

Ayer, A., Sanwald, J., Pillay, B. A., Meyer, A. J., Perrone, G. G., \& Dawes, I. W. (2013). Distinct redox regulation in sub-cellular compartments in response to various stress conditions in Saccharomyces cerevisiae. PLoS One, 8(6), e65240. doi: 10.1371/journal.pone. 0065240

Baumann, O., \& Walz, B. (2001). Endoplasmic reticulum of animal cells and its organization into structural and functional domains. Int Rev Cytol, 205, 149-214. doi: 10.1016/s00747696(01)05004-5

Bhaskar, A., Chawla, M., Mehta, M., Parikh, P., Chandra, P., Bhave, D., . . . Singh, A. (2014). Reengineering redox sensitive GFP to measure mycothiol redox potential of Mycobacterium tuberculosis during infection. PLOS Pathog, 10(1), e1003902. doi: 10.1371/journal.ppat.1003902

Birk, J., Meyer, M., Aller, I., Hansen, H. G., Odermatt, A., Dick, T. P., . . Appenzeller-Herzog, C. (2013). Endoplasmic reticulum: reduced and oxidized glutathione revisited. J Cell Sci, 126(Pt 7), 1604-1617. doi: 10.1242/jcs.117218

Brach, T., Soyk, S., Müller, C., Hinz, G., Hell, R., Brandizzi, F., \& Meyer, A. J. (2009). Non-invasive topology analysis of membrane proteins in the secretory pathway. Plant J, 57(3), 534-541. doi: 10.1111/j.1365-313X.2008.03704.x 
Burdakov, D., Petersen, O. H., \& Verkhratsky, A. (2005). Intraluminal calcium as a primary regulator of endoplasmic reticulum function. Cell Calcium, 38(3-4), 303-310. doi: 10.1016/j.ceca.2005.06.010

Coussin, F., Macrez, N., Morel, J. L., \& Mironneau, J. (2000). Requirement of ryanodine receptor subtypes 1 and 2 for $\mathrm{Ca}(2+)$-induced $\mathrm{Ca}(2+)$ release in vascular myocytes. J Biol Chem, 275(13), 9596-9603. doi: 10.1074/jbc.275.13.9596

Delic, M., Mattanovich, D., \& Gasser, B. (2010). Monitoring intracellular redox conditions in the endoplasmic reticulum of living yeasts. FEMS Microbiol Lett, 306(1), 61-66. doi: 10.1111/j.1574-6968.2010.01935.x

Ellgaard, L., \& Helenius, A. (2003). Quality control in the endoplasmic reticulum. Nat Rev Mol Cell Biol, 4(3), 181-191. doi: 10.1038/nrm1052

Ellgaard, L., \& Ruddock, L. W. (2005). The human protein disulphide isomerase family: substrate interactions and functional properties. EMBO Rep, 6(1), 28-32. doi: 10.1038/sj.embor.7400311

Ghaemmaghami, S., Huh, W. K., Bower, K., Howson, R. W., Belle, A., Dephoure, N., . . Weissman, J. S. (2003). Global analysis of protein expression in yeast. Nature, 425(6959), 737-741. doi: $10.1038 /$ nature02046

Glaumann, H., Bergstrand, A., \& Ericsson, J. L. (1975). Studies on the synthesis and intracellular transport of lipoprotein particles in rat liver. J Cell Biol, 64(2), 356-377. doi: 10.1083/jcb.64.2.356

Gutscher, M., Pauleau, A. L., Marty, L., Brach, T., Wabnitz, G. H., Samstag, Y., . . Dick, T. P. (2008). Real-time imaging of the intracellular glutathione redox potential. Nat Methods, 5(6), 553559. doi: $10.1038 /$ nmeth.1212

Hanson, G. T., Aggeler, R., Oglesbee, D., Cannon, M., Capaldi, R. A., Tsien, R. Y., \& Remington, S. J. (2004). Investigating mitochondrial redox potential with redox-sensitive green fluorescent protein indicators. J Biol Chem, 279(13), 13044-13053. doi: 10.1074/jbc.M312846200

Harding, H. P., Zhang, Y., Zeng, H., Novoa, I., Lu, P. D., Calfon, M., . . Ron, D. (2003). An integrated stress response regulates amino acid metabolism and resistance to oxidative stress. Mol Cell, 11(3), 619-633. doi: 10.1016/s1097-2765(03)00105-9

Hu, J., Dong, L., \& Outten, C. E. (2008). The redox environment in the mitochondrial intermembrane space is maintained separately from the cytosol and matrix. J Biol Chem, 283(43), 2912629134. doi: 10.1074/jbc.M803028200

Hwang, C., Sinskey, A. J., \& Lodish, H. F. (1992). Oxidized redox state of glutathione in the endoplasmic reticulum. Science, 257(5076), 1496-1502. doi: 10.1126/science.1523409

Inui, M., Saito, A., \& Fleischer, S. (1987). Purification of the ryanodine receptor and identity with feet structures of junctional terminal cisternae of sarcoplasmic reticulum from fast skeletal muscle. J Biol Chem, 262(4), 1740-1747.

Kanekura, K., Ishigaki, S., Merksamer, P. I., Papa, F. R., \& Urano, F. (2013). Establishment of a system for monitoring endoplasmic reticulum redox state in mammalian cells. Lab Invest, 93(11), 1254-1258. doi: 10.1038/labinvest.2013.112

Kasahara, E., Lin, L. R., Ho, Y. S., \& Reddy, V. N. (2005). SOD2 protects against oxidation-induced apoptosis in mouse retinal pigment epithelium: implications for age-related macular degeneration. Invest Ophthalmol Vis Sci, 46(9), 3426-3434. doi: 10.1167/iovs.05-0344

Kirstein, J., Morito, D., Kakihana, T., Sugihara, M., Minnen, A., Hipp, M. S., . . Morimoto, R. I. (2015). Proteotoxic stress and ageing triggers the loss of redox homeostasis across cellular compartments. Embo j, 34(18), 2334-2349. doi: 10.15252/embj.201591711

Kozutsumi, Y., Segal, M., Normington, K., Gething, M. J., \& Sambrook, J. (1988). The presence of malfolded proteins in the endoplasmic reticulum signals the induction of glucose-regulated proteins. Nature, 332(6163), 462-464. doi: 10.1038/332462a0 
Lai, F. A., Erickson, H. P., Rousseau, E., Liu, Q. Y., \& Meissner, G. (1988). Purification and reconstitution of the calcium release channel from skeletal muscle. Nature, 331(6154), 315319. doi: $10.1038 / 331315 \mathrm{a} 0$

Lohman, J. R., \& Remington, S. J. (2008). Development of a family of redox-sensitive green fluorescent protein indicators for use in relatively oxidizing subcellular environments. Biochemistry, 47(33), 8678-8688. doi: 10.1021/bi800498g

Merksamer, P. I., Trusina, A., \& Papa, F. R. (2008). Real-time redox measurements during endoplasmic reticulum stress reveal interlinked protein folding functions. Cell, 135(5), 933947. doi: 10.1016/j.cell.2008.10.011

Montero, D., Tachibana, C., Rahr Winther, J., \& Appenzeller-Herzog, C. (2013). Intracellular glutathione pools are heterogeneously concentrated. Redox Biol, 1(1), 508-513. doi: 10.1016/j.redox.2013.10.005

Morgan, B., Ezerina, D., Amoako, T. N., Riemer, J., Seedorf, M., \& Dick, T. P. (2013). Multiple glutathione disulfide removal pathways mediate cytosolic redox homeostasis. Nat Chem Biol, 9(2), 119-125. doi: 10.1038/nchembio.1142

$\emptyset$ stergaard, H., Tachibana, C., \& Winther, J. R. (2004). Monitoring disulfide bond formation in the eukaryotic cytosol. J Cell Biol, 166(3), 337-345. doi: 10.1083/jcb.200402120

Romero-Aristizabal, C., Marks, D. S., Fontana, W., \& Apfeld, J. (2014). Regulated spatial organization and sensitivity of cytosolic protein oxidation in Caenorhabditis elegans. Nat Commun, 5, 5020. doi: $10.1038 /$ ncomms 6020

Schuiki, I., Zhang, L., \& Volchuk, A. (2012). Endoplasmic reticulum redox state is not perturbed by pharmacological or pathological endoplasmic reticulum stress in live pancreatic $\beta$-cells. PLOS One, 7(11), e48626. doi: 10.1371/journal.pone.0048626

Sitia, R., \& Meldolesi, J. (1992). Endoplasmic reticulum: a dynamic patchwork of specialized subregions. Mol Biol Cell, 3(10), 1067-1072. doi: 10.1091/mbc.3.10.1067

Takeshima, H. (2002). Intracellular Ca2+ store in embryonic cardiac myocytes. Front Biosci, 7, d16421652. doi: $10.2741 /$ takeshim

van Lith, M., Tiwari, S., Pediani, J., Milligan, G., \& Bulleid, N. J. (2011). Real-time monitoring of redox changes in the mammalian endoplasmic reticulum. J Cell Sci, 124(Pt 14), 2349-2356. doi: $10.1242 /$ jcs. 085530

Vidugiriene, J., \& Menon, A. K. (1993). Early lipid intermediates in glycosyl-phosphatidylinositol anchor assembly are synthesized in the ER and located in the cytoplasmic leaflet of the ER membrane bilayer. J Cell Biol, 121(5), 987-996. doi: 10.1083/jcb.121.5.987

Wang, Y., Branicky, R., Noë, A., \& Hekimi, S. (2018). Superoxide dismutases: Dual roles in controlling ROS damage and regulating ROS signaling. 217(6), 1915-1928. doi: 10.1083/jcb.201708007

Zeeshan, H. M., Lee, G. H., Kim, H. R., \& Chae, H. J. (2016). Endoplasmic Reticulum Stress and Associated ROS. Int J Mol Sci, 17(3), 327. doi: 10.3390/ijms17030327

Zheng, H. Q., \& Staehelin, L. A. (2001). Nodal endoplasmic reticulum, a specialized form of endoplasmic reticulum found in gravity-sensing root tip columella cells. Plant Physiol, 125(1), 252-265. doi: 10.1104/pp.125.1.252 


\section{Figure legends}

Figure 1: roGFP2 is localized in Endoplasmic reticulum in the generated eroGFP transgenic line. (A) A schematic representation of the construct and strategy to make the transgenic lines, where roGFP2 was targeted to cytosol and ER both, and roGFP1/iE to ER. The construct consisted of actin promoter for ubiquitous expression and flanked by tol 2 sites on which transposase works. The lines are named according to the zfin nomenclature. (B) Amino acid (aa) sequence alignment of GRP78 from zebrafish and Human is shown. MEGA was used for alignment and to find out the ER targeting signal sequence HSP70 and Hsp8b was used as control. Dotted red box shows the 16 aa taken for adding before roGFP2 probes for ER targeting. For ERroGFPiE line, 26 aa were taken.(C) A schematic to show generation of primary cell culture from zebrafish embryos (D) fluorescence images of primary culture made from $1 \mathrm{dpf}$ zebrafish embryos showing localization of roGFP in eroGFP (left panel) and cyroGFP (middle panel) line. Images on bottom in these panels show zoomed area as shown in the images on top. Right panel shows roGFP signal (top) and ERTracker (middle), and merge (bottom) in eroGFP line respectively, where ER-Tracker is used to assess location of localization of GFP in ER (confocal microscope, 63X objective, 4X

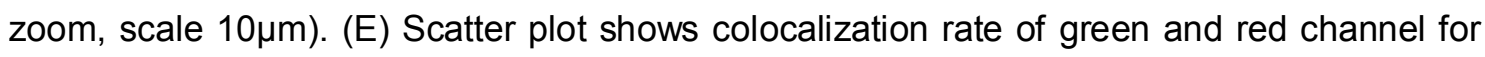
eroGFP line and each dot represents one ROI. Two tailed Student's $t$ test with unequal variance was done to test significance, ${ }^{* * * *} p$-value $<0.0001$. (Dpf- days post fertilization, ER- endoplasmic reticulum, mito- mitochondria, $\mathrm{ROI}$ region of interest)

Figure 2: roGFP transgenic lines for ER do not exhibit perturbations in homeostasis. (A) Protein lysates from 39 embryos were loaded on a SDS gel and the transfer was done. Western blots show expression of Hspa5 (GRP78) and Sod2 in the ER transgenic lines and wild type along with $1 \mathrm{mM}$ DTT treated embryos ( $8 \mathrm{hrs}$ ) taken as a positive control. GFP was also probed and actin was used as a loading control. (B) and (C) The graph shows quantification of Hspa5 and Sod2 expression w.r.t. actin respectively which was done using ImageJ. (D) 3 live embryos at $5 \mathrm{dpf}$ were put in each well of a 96 well plate. Water was removed to an extent that embryos have some water to survive and DCFDA dye was added. Incubation was done for $2 \mathrm{hrs}$ and readings were taken. Wells with embryos incubated in $\mathrm{H}_{2} \mathrm{O}_{2}$ was used as a positive control. 12 wells were used for one replicate, so each replicate had 36 embryos. (WT- wild type, w.r.t- with respect to)

Figure 3: Primary culture cells from roGFP transgenic lines are sensitive to chemical perturbations where tunicamycin $(\mathrm{Tm})$ does not lead to reducing redox state. (A) Primary cells from $1 \mathrm{dpf}$ embryos were adhered, grown overnight and 6 and $10 \mu \mathrm{g} / \mathrm{ml} \mathrm{Tm}$ treatment was given for $8 \mathrm{hrs}$. Western blots show expression of GRP78 and GRP94 as ER stress markers, where actin was used as a loading control and GFP was also probed. (B) 
Cells were treated with $6 \mu \mathrm{g} / \mathrm{ml}$ Tm for $8 \mathrm{hrs}$ and then redox imaging i.e., first excited at 405 $\mathrm{nm}$ and emission collected at 500 to $540 \mathrm{~nm}$, same was done for $488 \mathrm{~nm}$, using confocal microscope. Raw images were then analyzed by MATLAB script to calculate pixel wise Ratio-2 values, as described in materials and methods. The pixels were divided into 3 bins according to the values obtained and given RGB scale. R1 is the boundary defined as 1 standard deviation away from mean eroGFP value while R2 is 1 standard deviation less than mean cyroGFP value. Ratio-2 below R1 were given red (oxidizing), between R1 and R2 were green (intermediate) and higher than R2 were blue color (reducing). (C) Along with Tm treatment, cells were also treated with $10 \mathrm{mM}$ DTT and imaging was started immediately. Images represent maximum projection images of untreated, $10 \mathrm{mM}$ DTT and $6 \mu \mathrm{g} / \mathrm{ml} \mathrm{Tm}$ treated cells from left to right respectively (63X objective, zoom 4). (D) Ratio-2 values are plotted for each of these conditions. Each line represents experiment mean Ratio-2 for each treatment (SET1), done on one day and it was repeated thrice. $\mathrm{N}$ is variable in each condition and contains value from at least 6 fields. (BF- bright field)

Figure 4: Elucidation of autofluorescence signals from a WT embryo. (A) WT embryo lacking any roGFP expression was anaesthetized, mounted in lateral position, excited at 405 $\mathrm{nm}$ and emission scan was taken from 422 to $593 \mathrm{~nm}$ in order to know the autofluorescence signals (upper panel). Readings were taken at interval of every $3 \mathrm{~nm}$ and the graph was plotted. Three lines represent three individual embryos. Similarly, emission scan was taken for $1 \mu \mathrm{M}$ roGFP2 solution at same settings (lower panel). (B) Embryos were anaesthetized, mounted in agar in lateral position and imaging was done with excitation at $405 \mathrm{~nm}$ and emission was collected from 440-480 nm. Representative autofluorescence signal from one WT embryo is a maximum projection image adjusted with ImageJ.

Figure 5: Representative cross sections and regions of interests marked on GFP fluorescence images of an embryo. (A) The left image shows the way embryo was imaged with yellow plane showing the region where optical sectioning was done to show the redox ratios inside the embryo. Middle and right image indicates the way embryo was oriented (rotated) to $30^{\circ}$ and $70^{\circ}$ respectively, also indicated by the arrow, making yellow disc visible in the lower image. (B) to (D) Images show GFP signal of an embryo representing the rotation angles to show redox ratios in the subsequent figures. Cross section at these positions remains similar for further figures. (B) All images in the panel show the region from where optical sectioning was done and $(C)$ panel shows the GFP signal in the oriented image of embryo sectioned at that region. (C) All images in the panel from left to right shows labelling of major regions, forebrain, midbrain, hindbrain, muscle neurocoel and notochord, which become visible after sectioning done at the places indicated and embryo rotated at $30^{\circ}$. (D) All images in the panel shows same regions when embryo is rotated at 
$90^{\circ}$. White line in each right panel image indicates the lateral line which will divide the embryo in left and right halves. (Confocal microscope, 10X objective)

Figure 6: Redox status of cytosol is also heterogeneous in zebrafish embryos (cyroGFP line). $3 \mathrm{dpf}$ live eroGFP embryos were imaged and analyzed for getting redox ratios. Redox ratios were obtained after image analysis where pixel wise Ratio-2 were calculated using MATLAB. (A) Ratio-2 values for cyroGFP lines were plotted to see ratio-2 distribution with eroGFP Ratio-2 values as control. R3 and R4 boundaries were set to define color scale where R3 is median of ratio-2 from eroGFP embryo and R4 is one standard deviation from eroGFP median. Ratio-2 values below R3 were oxidizing and given red color. Ratios higher than R4 were reducing and given blue color while intermediate values between R3 and R4 were green. (B) Ratio-2 values of four embryos depicted by embryo 1, 2, 3, and 4. (C) Left and right panel show GFP fluorescence with optical section and analyzed Ratio-2 images of a representative embryo respectively. First, second, third and fourth panel are images obtained after optical sectioning and embryo rotation to $90^{\circ}$. Yellow and white arrow indicates forebrain and hindbrain respectively in (B) and (C). (D) Log-2 values of Ratio-2 were plotted against area normalized frequency values. Red colored circle represents Ratio-2 values for all embryos and black solid line is obtained after gaussian fit of the Ratio- 2 values. The black arrow shows regions where oxidizing redox ratios are above the fitted black solid line (E) Higher magnification (40X) images of a representative embryo shows the head region (behind the eye), initial trunk (behind the yolk) and trunk regions (muscle; myotomes) from top to bottom while left and right are Ratio-2 and BF images respectively. (BF-bright field, confocal microscope, 10X and 40X objectives)

Figure 7: redox state of ER is different in some regions of zebrafish embryos (eroGFP line). 3 dpf live eroGFP embryos were molded and imaged for getting redox status. Ratio-2 were obtained after image analysis where pixel wise ratios were calculated using MATLAB. (A) Ratio-2 values of eroGFP embryos were plotted to get the distribution and define color scale with cyroGFP embryo as control. R5 and R6 boundaries were defined where R6 is median of cyroGFP embryo and R5 is one standard deviation lower than cyroGFP median. For color coding, pixels below R5 and higher than R6 were given red (oxidizing) and blue (reducing) color respectively. (B) Embryos images showing ratio-2 values of four embryos separately. (C) Left and right panel show GFP fluorescence with optical sections and analyzed Ratio-2 images respectively. First, second, third and fourth panel from top to bottom show the ratio-2 images sectioned at the indicated positions. (D) Log-2 values of ratio-2 were plotted against frequency with area normalization where red circles are real calculated values while black solid lines are fitted values. Black arrow indicates the real Ratio-2 values above the fitted data. (Confocal microscope, 10X objectives) 
Figure 8: redox state of ER is better visualized using ERroGFPiE line with more evident reducing regions. $3 \mathrm{dpf}$ live ERroGFPiE embryos were imaged and analyzed for redox (Ratio-iE) values. The roGFPiE solutions treated with $10 \mathrm{mM}$ DTT or $10 \mathrm{mM}$ diamide were also imaged using same settings. (A) log-2 values of Ratio-iE distribution for ERroGFPiE embryos were plotted against frequency (area normalized) and line represents mean Ratio-iE from embryos. The Ratio-iE can be divided into three normal distributions which are shown by three smaller peaks where red and blue line represents the Ratio-iE of fully oxidized (diamide treated) and reduced (DTT treated) roGFPiE solutions respectively. (B) The log-2 Ratio-iE distribution for ERroGFPiE embryos was binned in three groups using reduced and oxidized Ratio-iE values. Ratios lower than the median of oxidized roGFPiE solution ( $R 7$ boundary) were oxidizing and given red color. The values higher than the median of reduced roGFPiE solution (R8 boundary) were given blue color (reducing regions) and intermediate values between R7 and R8 were green. (C) Ratio-iE images of four roGFPiE embryos are shown suing the described color scale where white arrow shows the reducing patches in the forebrain. (D) Ratio-iE images obtained after optical sectioning at forebrain (left panel) and midbrain (right panel) regions followed by rotation of the embryos to $0 \square$ are shown. White arrow indicates reducing patches in the base of forebrain and beginning of the midbrain in three independent embryos (top to bottom). (E) Ratio-iE images of the embryos in another $Z$ plane showing reduced heart walls indicated using white arrows. (Confocal microscope, 10X objective)

\section{Table Legend.}

Table 1. Properties of the different peaks of Ratio-iE as obtained from the ERroGFP. iE embryos. $\log _{2}$ of Ratio-iE of each of the embryos (Embryo 1 to Embryo 4 ) are shown in the first 4 columns. The values are the peak values (means) for each of the peaks (oxidized, reduced, and intermediate). In the next 4 columns are shown the redox potential calculated from Ratio-2 based on the published redox potential of roGFP-iE for each of the embryo. The redox potential for the fully oxidized and reduced peak cannot be calculated and are left as NA. The percentage of total pixels that are present in each of the peaks (oxidized, reduced and intermediate) are given in the next four columns (one column for each of the embryos). 
Figure 1
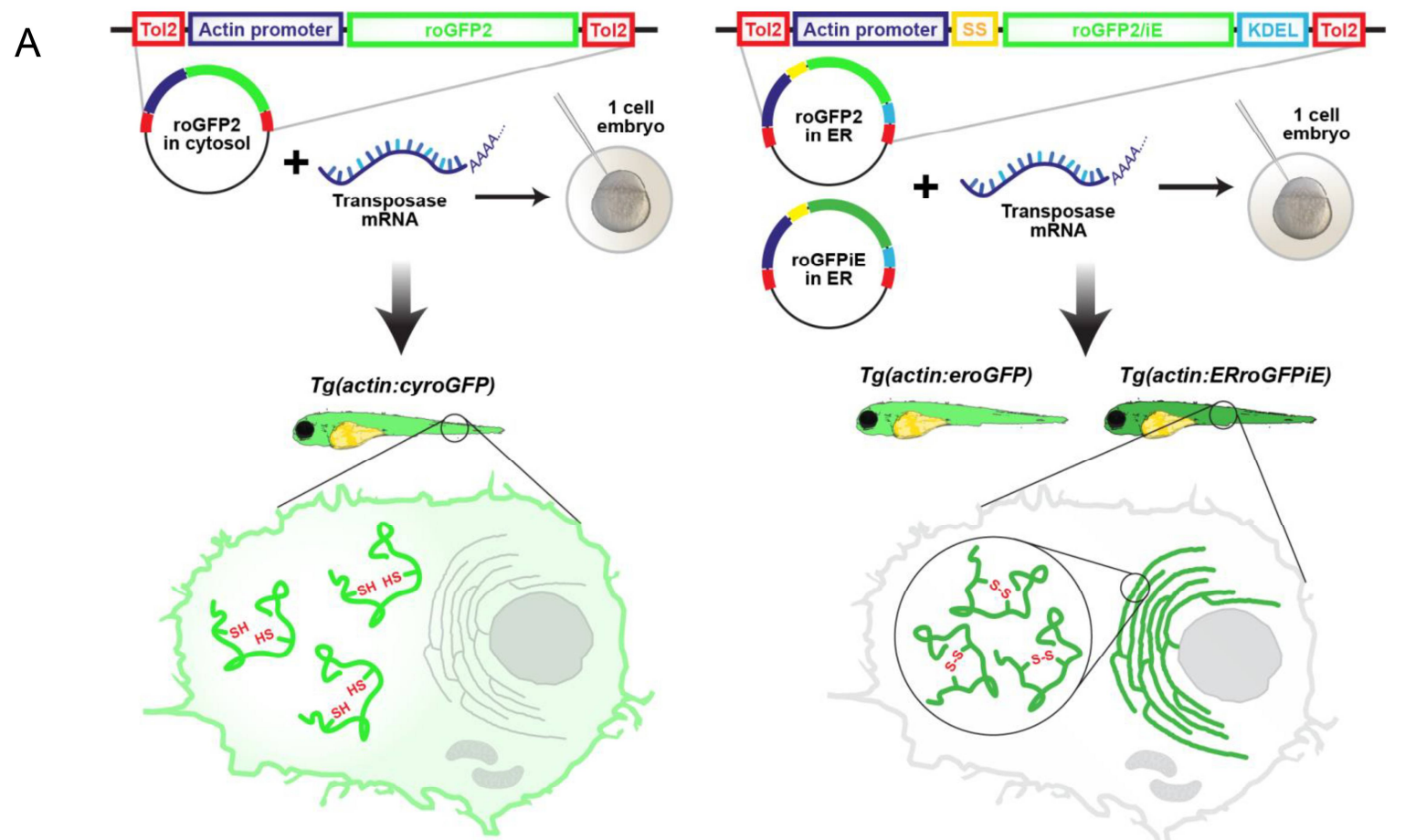

B

Homo sapiens_GRP78 MK L S L V A A M L L L L S A A R A E E E D K K E D V G T V V G Danio rerio_Hspa5 Homo sapiens_HSP70 Danio rerio_Hsp8b

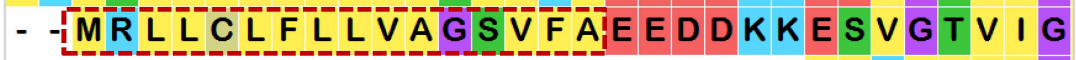

\section{Danio rerio Hsp}

C

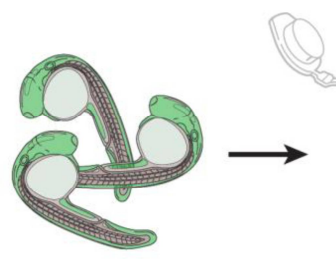

1 dpf embryos

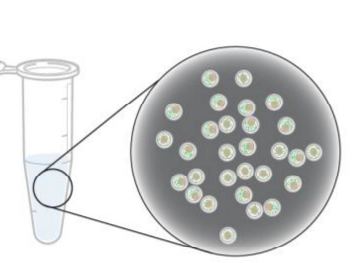

Deyolking, trypsinization and adhering

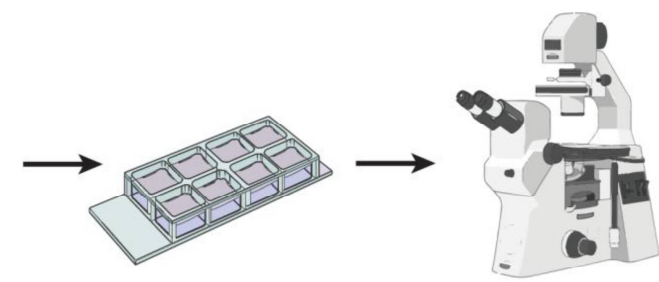

Grown overnight

Imaging

D
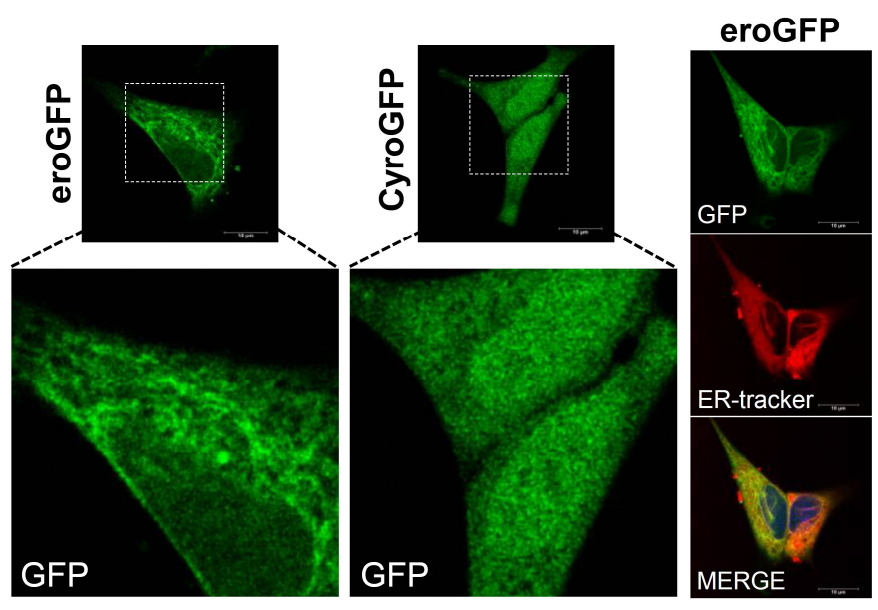

$E$

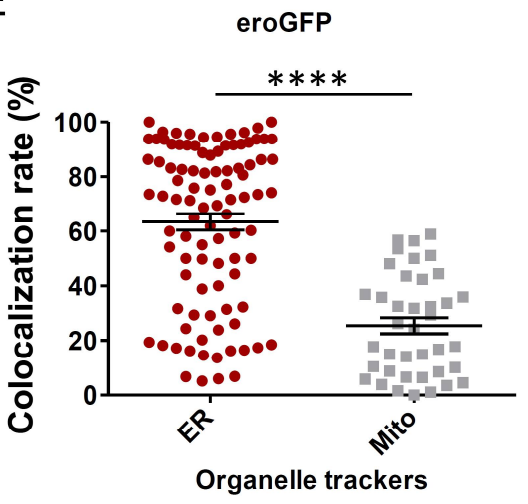


Figure 2

A

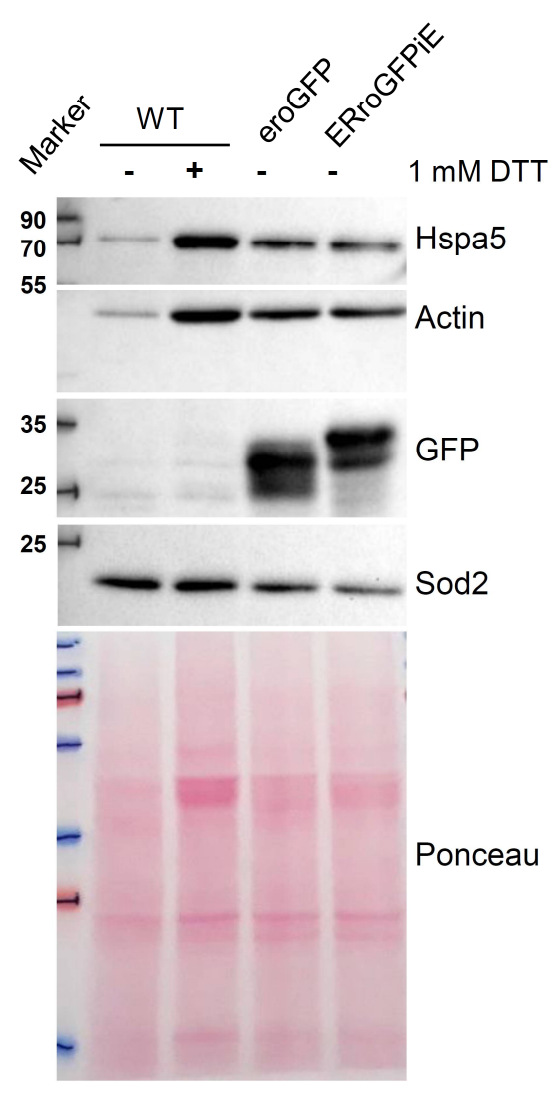

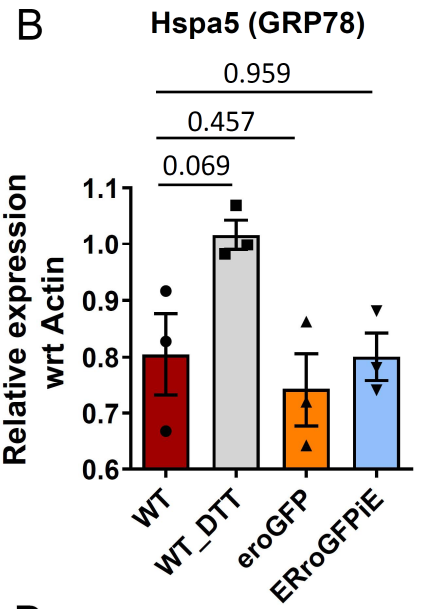

C Sod2

D Embryos in 96 well plate; 3 embryos/well

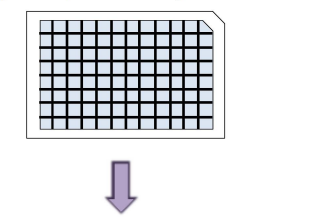

2.5 mM DCFDA; 2 hours incubation<smiles>C1=CC=C1</smiles>

Readings taken
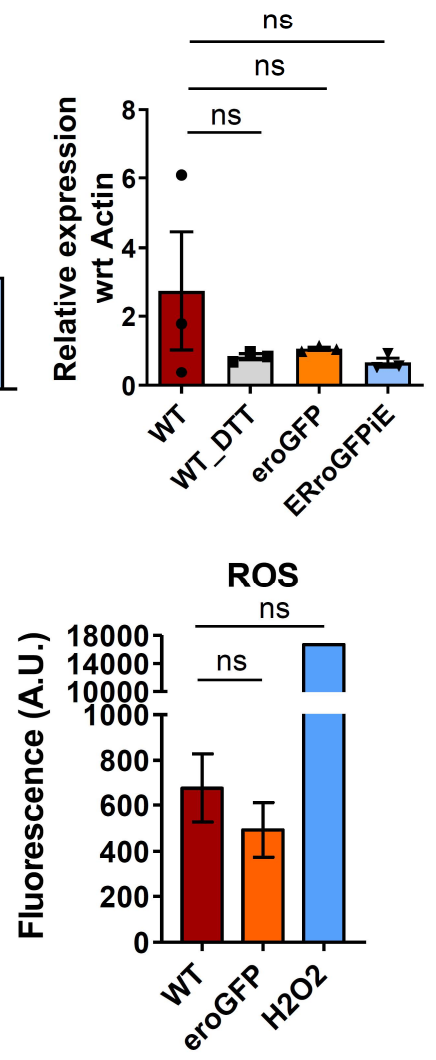
Figure 3

A

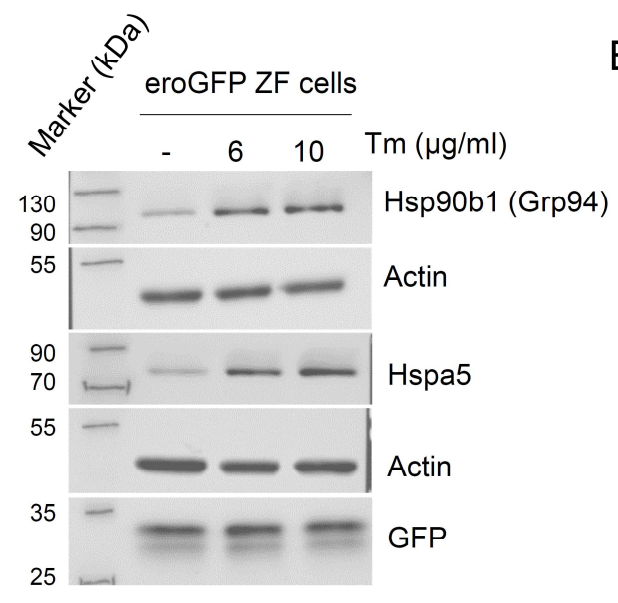

C

C

0
$\frac{1}{0}$
$\frac{0}{0}$

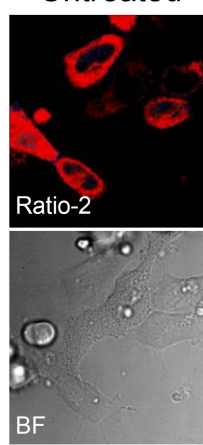

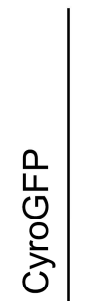

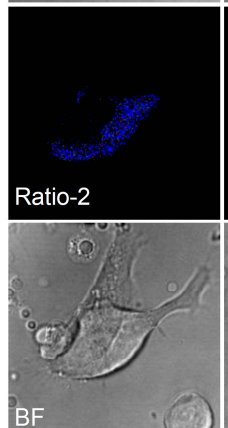

B

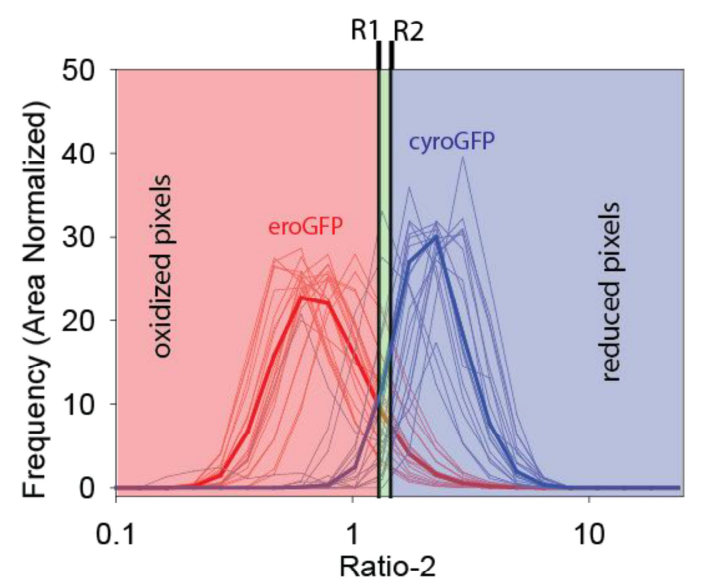

D

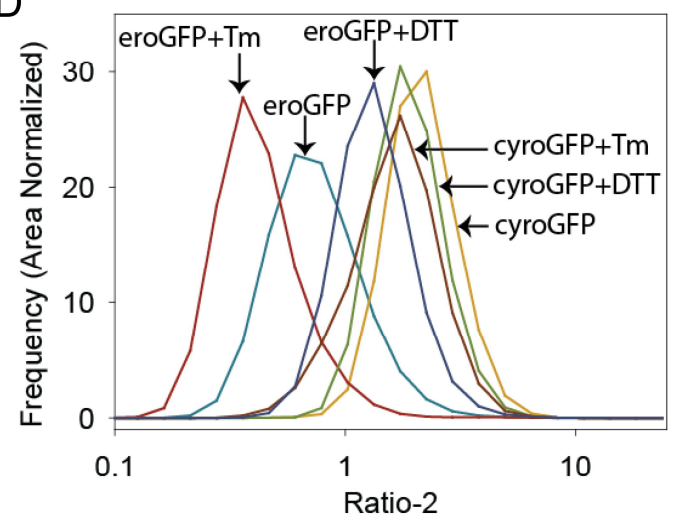


bioRxiv preprint doi: https://doi.org/10.1101/2022.02.12.480199; this version posted February 12, 2022. The copyright holder for this preprint (which was not certified by peer review) is the author/funder. All rights reserved. No reuse allowed without permission.

Figure 4

A

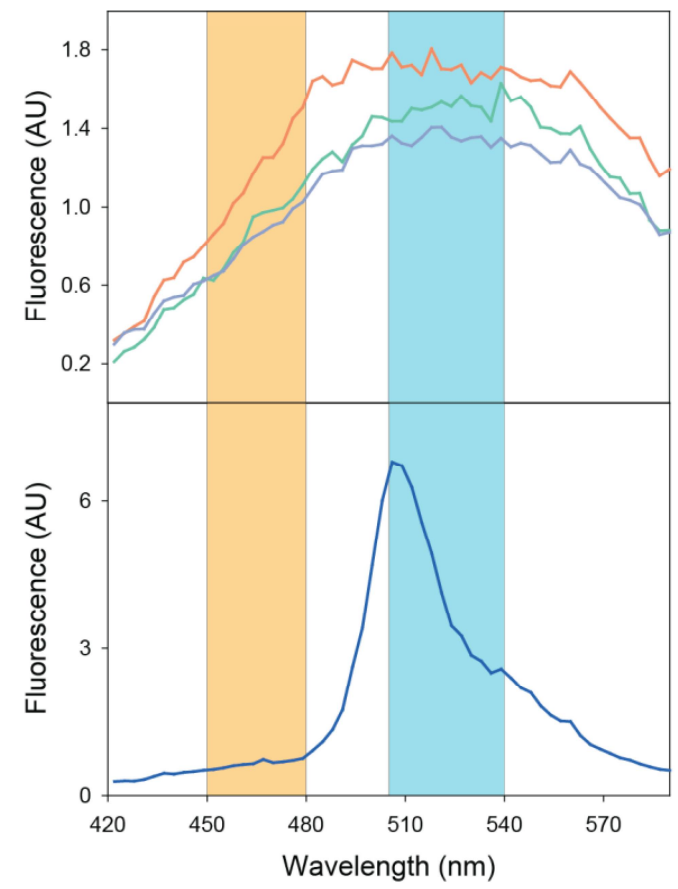

B

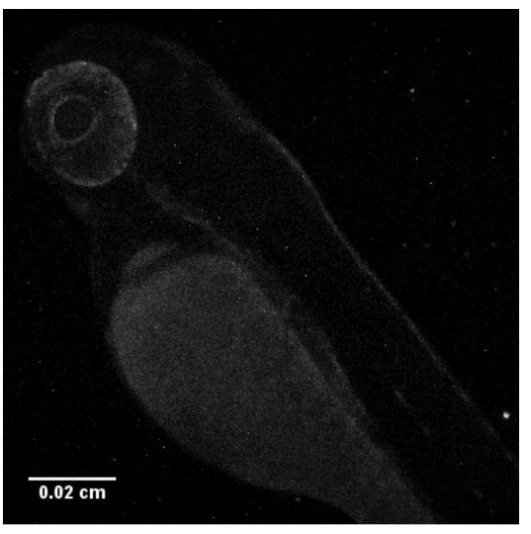


bioRxiv preprint doi: https://doi.org/10.1101/2022.02.12.480199; this version posted February 12, 2022. The copyright holder for this preprint (which was not certified by peer review) is the author/funder. All rights reserved. No reuse allowed without permission.

Figure 5
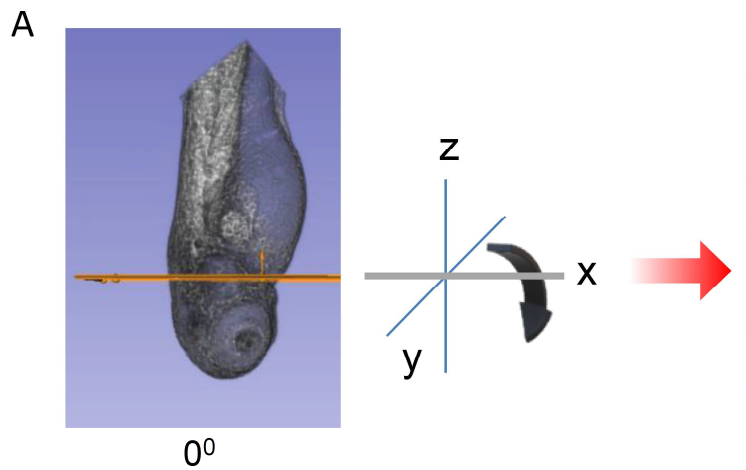

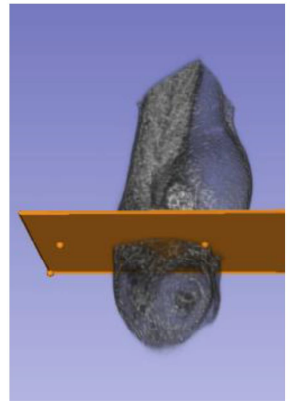

$30^{\circ}$

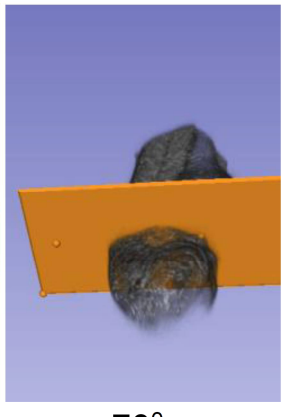

$70^{\circ}$

B
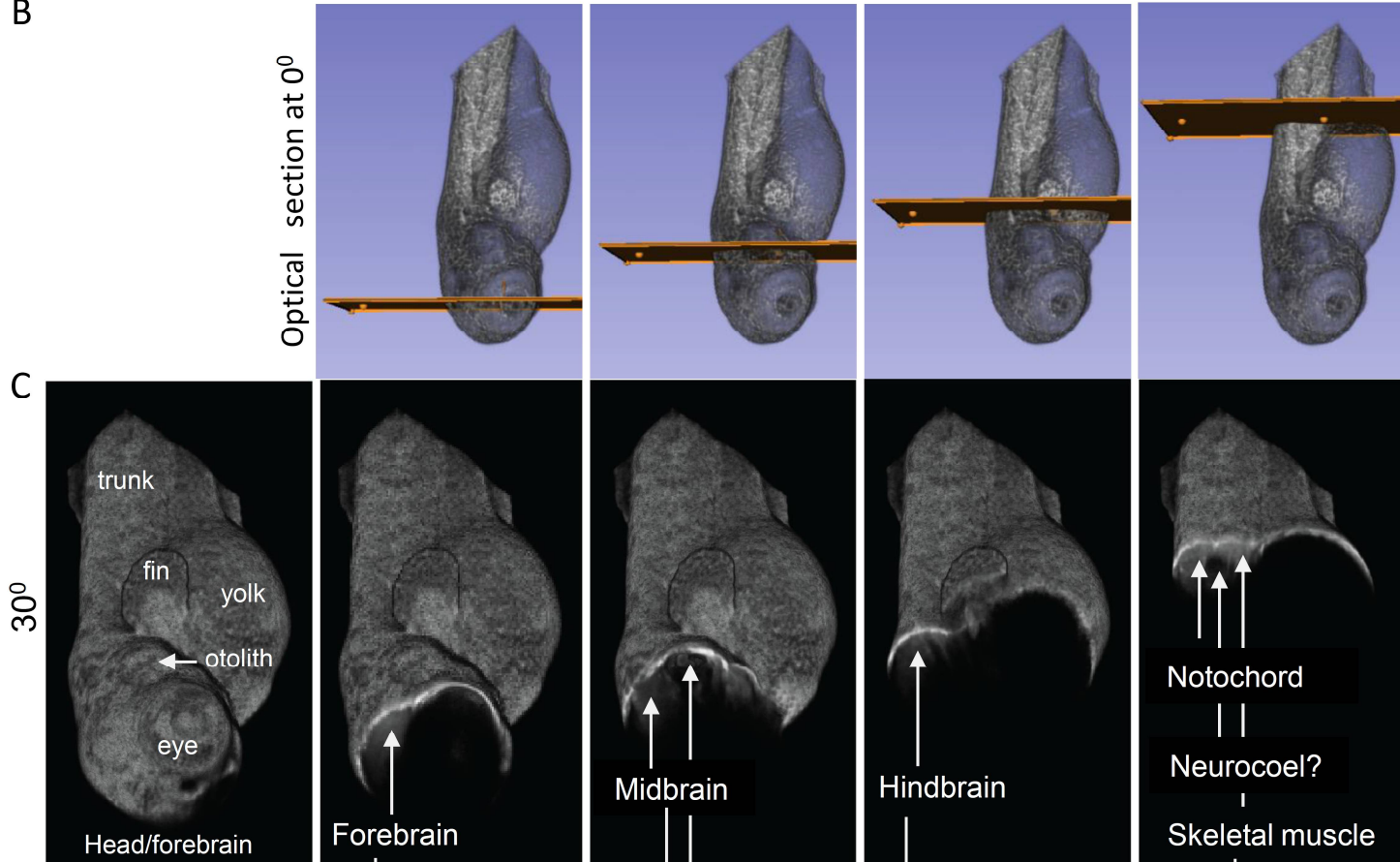

Notochord

| |

Neurocoel?

I

Skeletal muscle

D
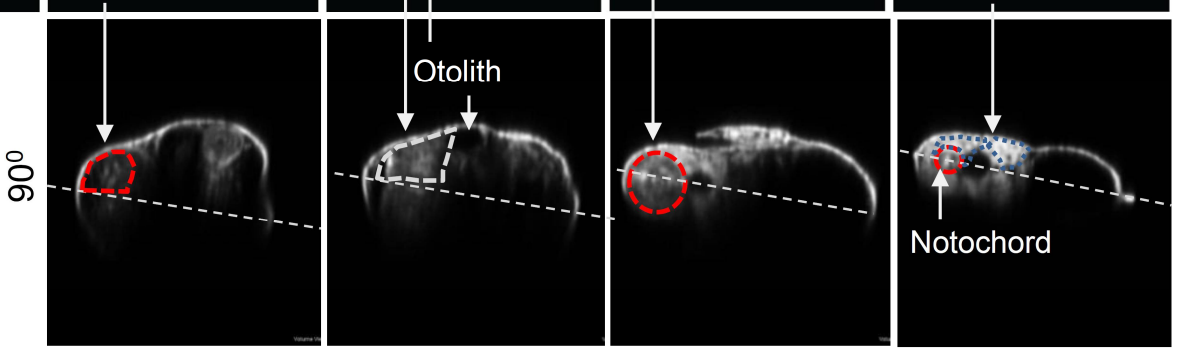
bioRxiv preprint doi: https://doi.org/10.1101/2022.02.12.480199; this version posted February 12, 2022. The copyright holder for this preprint (which was not certified by peer review) is the author/funder. All rights reserved. No reuse allowed without permission.

Figure 6
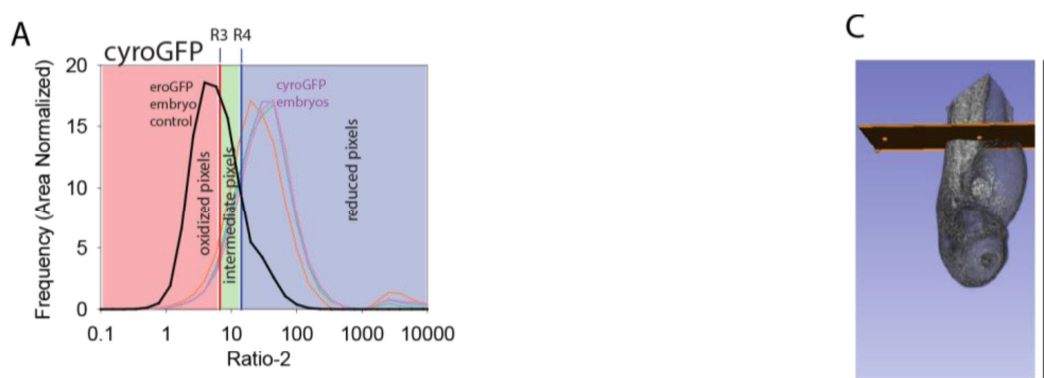

B
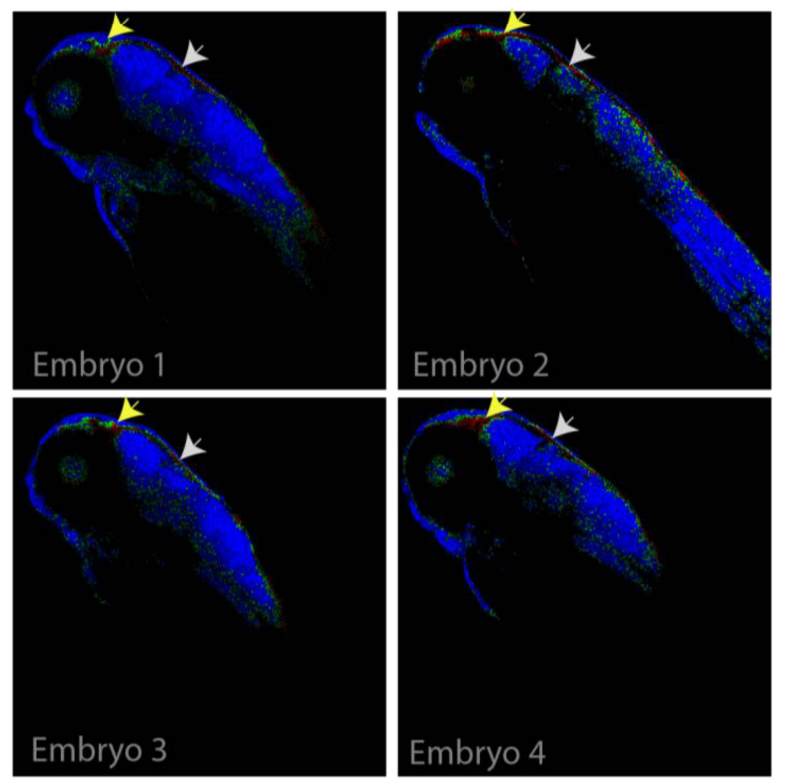

D
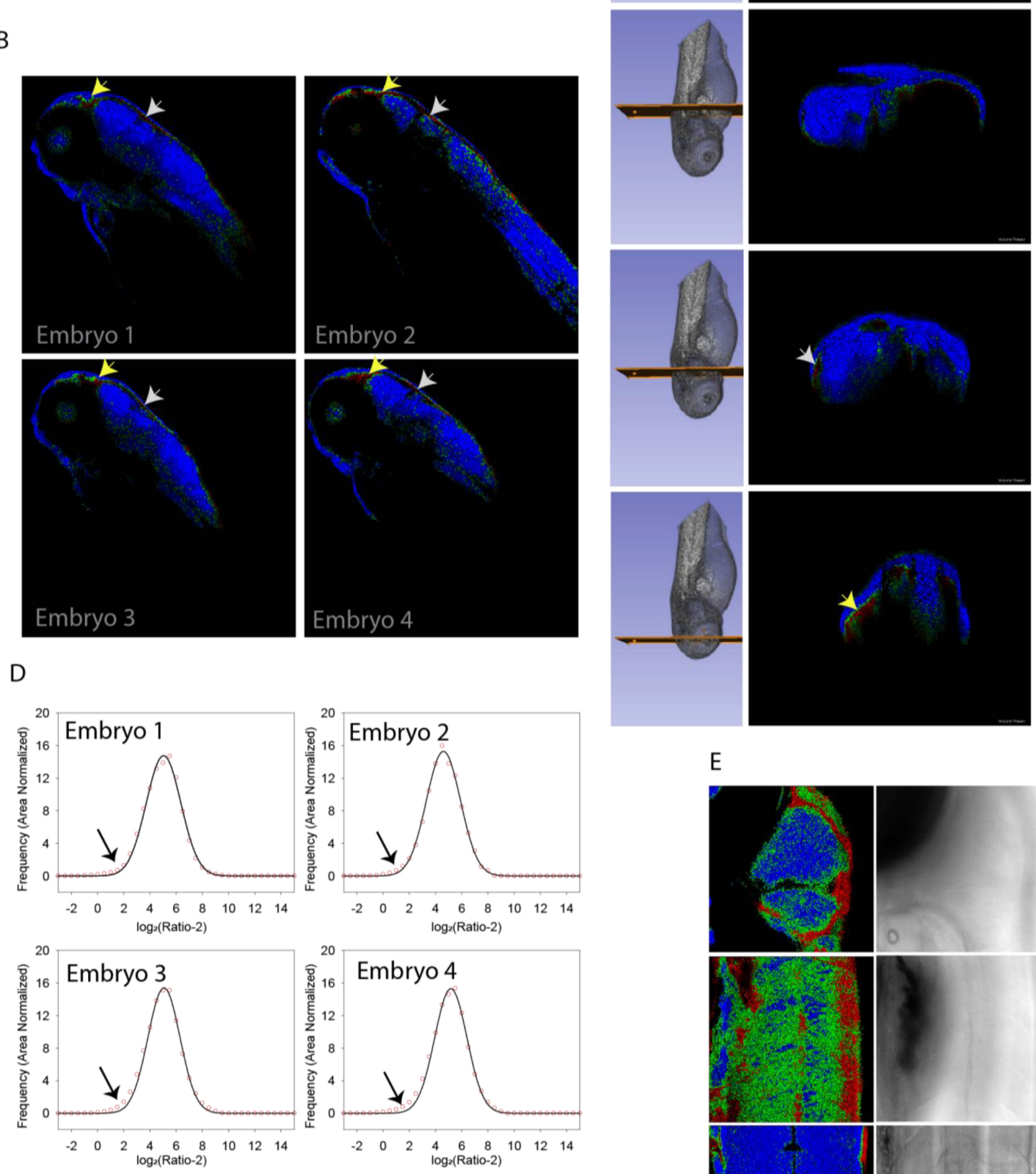

E

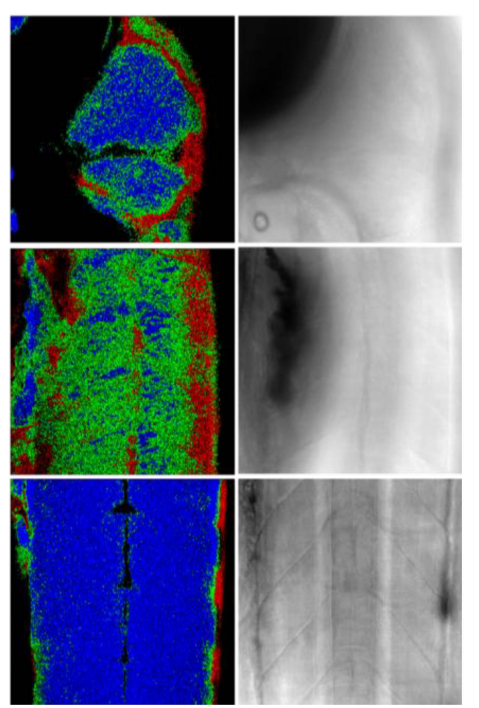


bioRxiv preprint doi: https://doi.org/10.1101/2022.02.12.480199; this version posted February 12, 2022. The copyright holder for this preprint (which was not certified by peer review) is the author/funder. All rights reserved. No reuse allowed without permission.

Figure 7

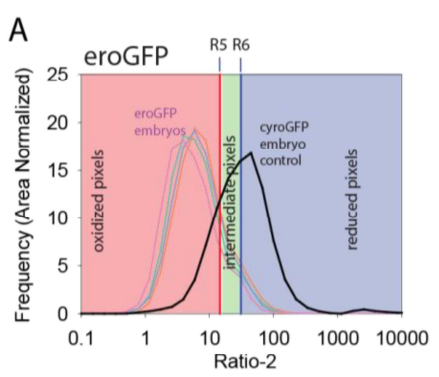

C
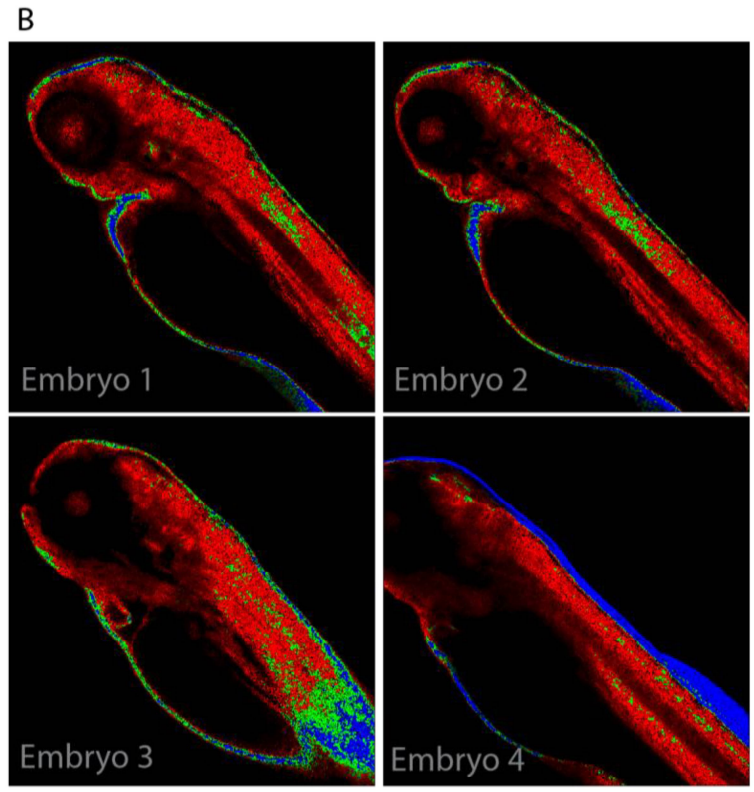

D
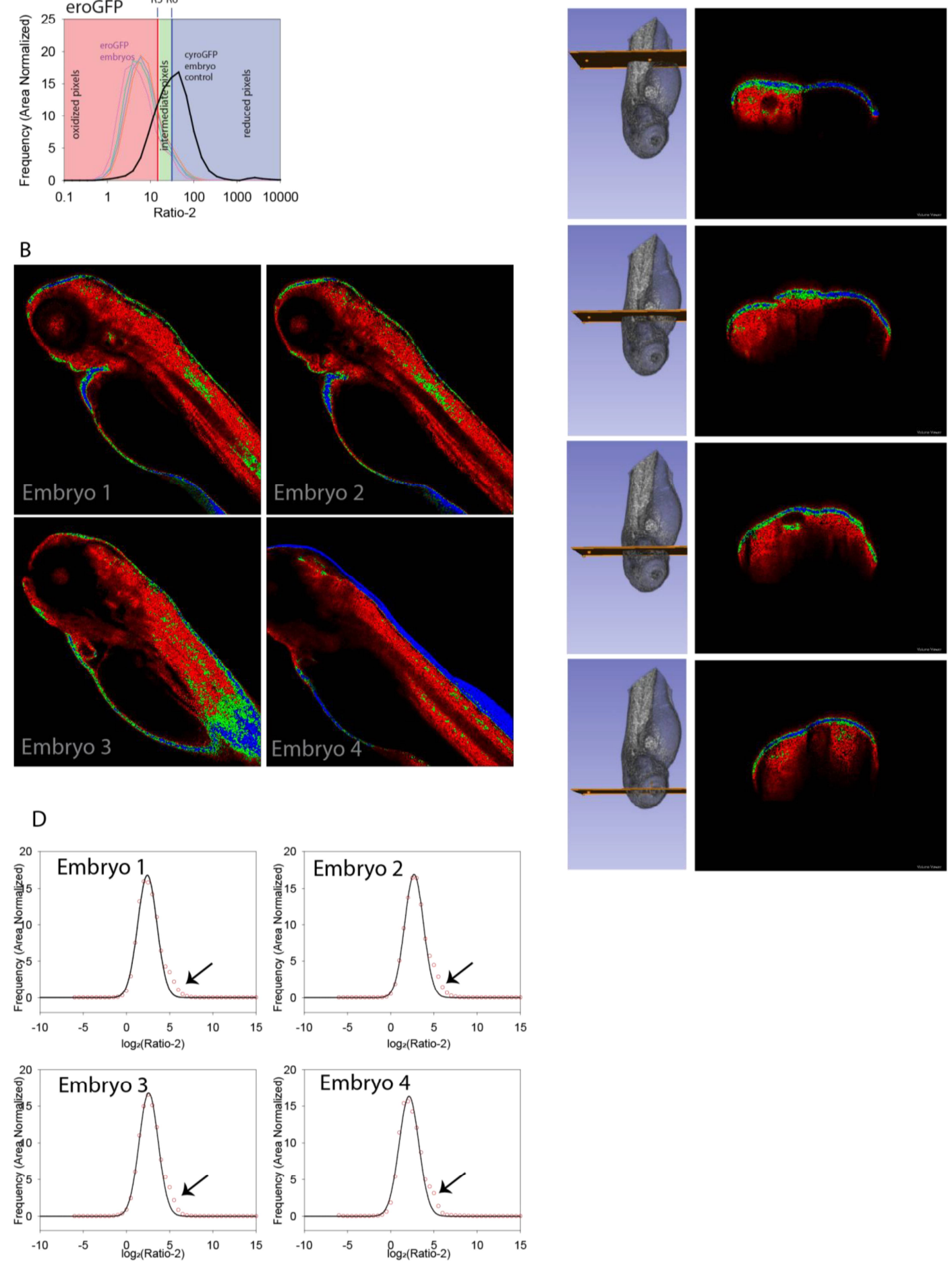
bioRxiv preprint doi: https://doi.org/10.1101/2022.02.12.480199; this version posted February 12, 2022. The copyright holder for this preprint (which was not certified by peer review) is the author/funder. All rights reserved. No reuse allowed without permission.

Figure 8

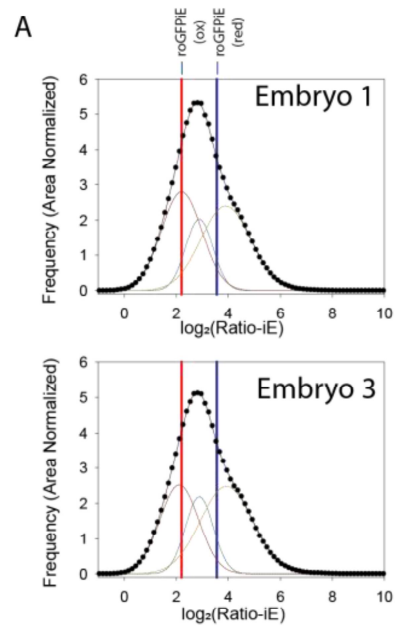

D
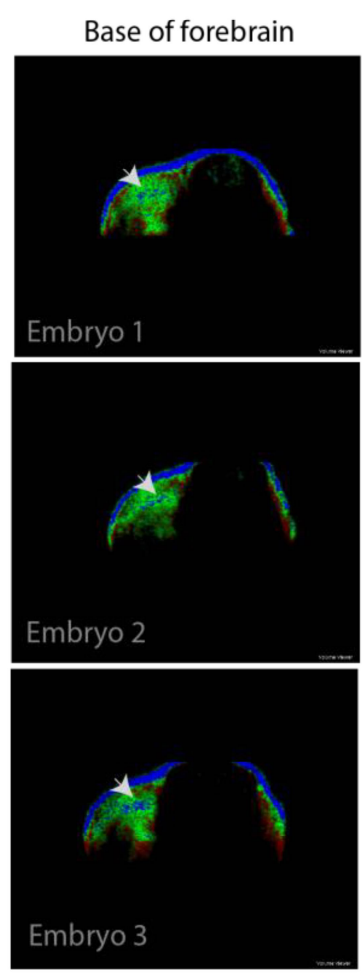
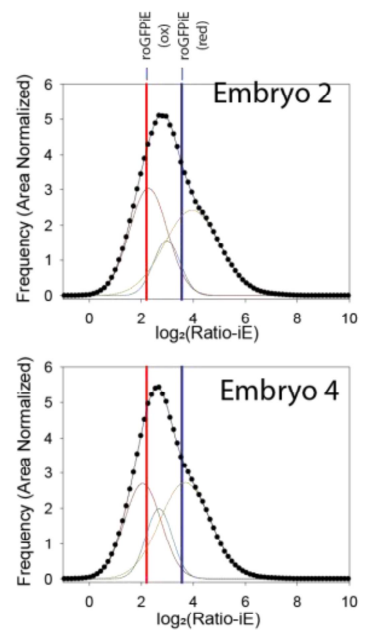

Beginning of midbrain
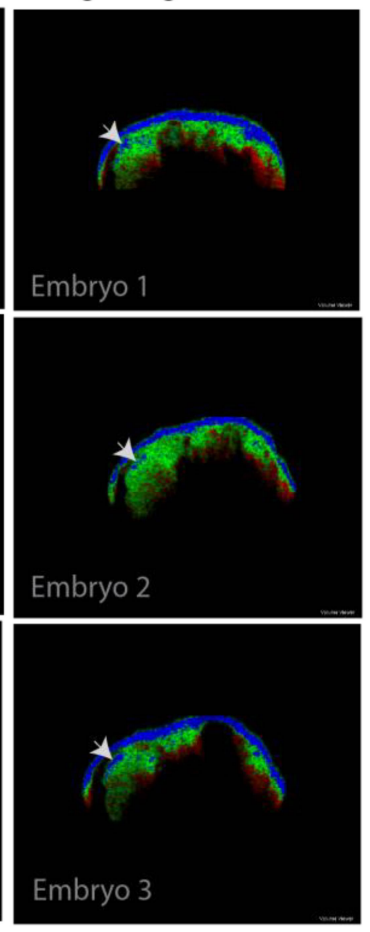

B

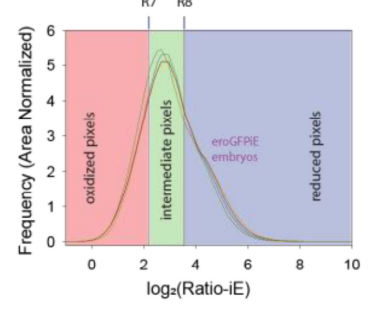

C

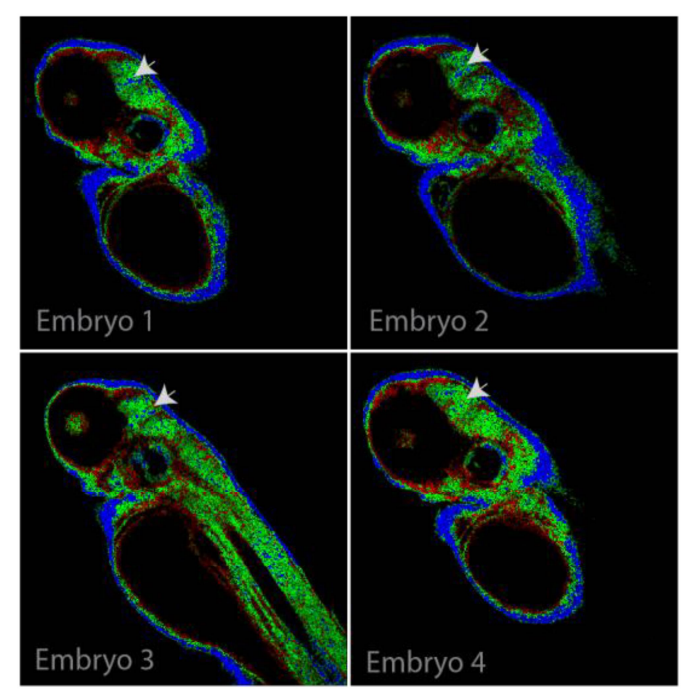

E
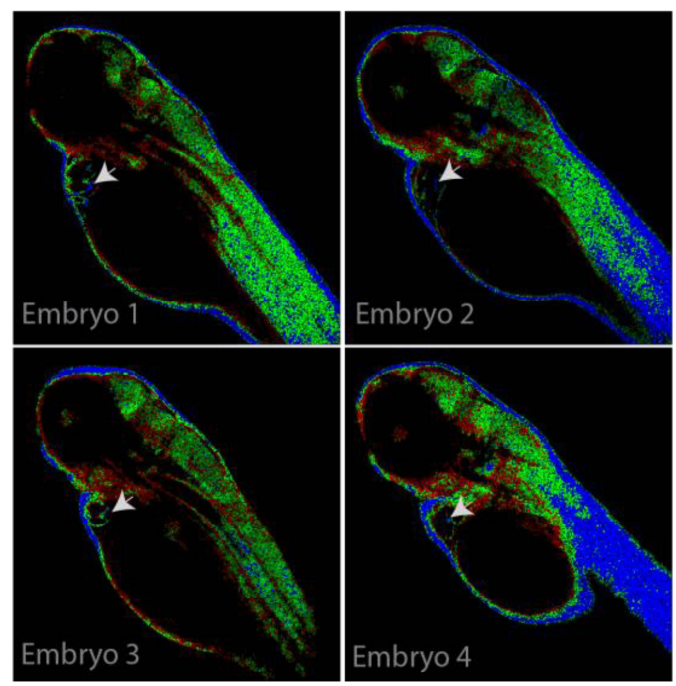


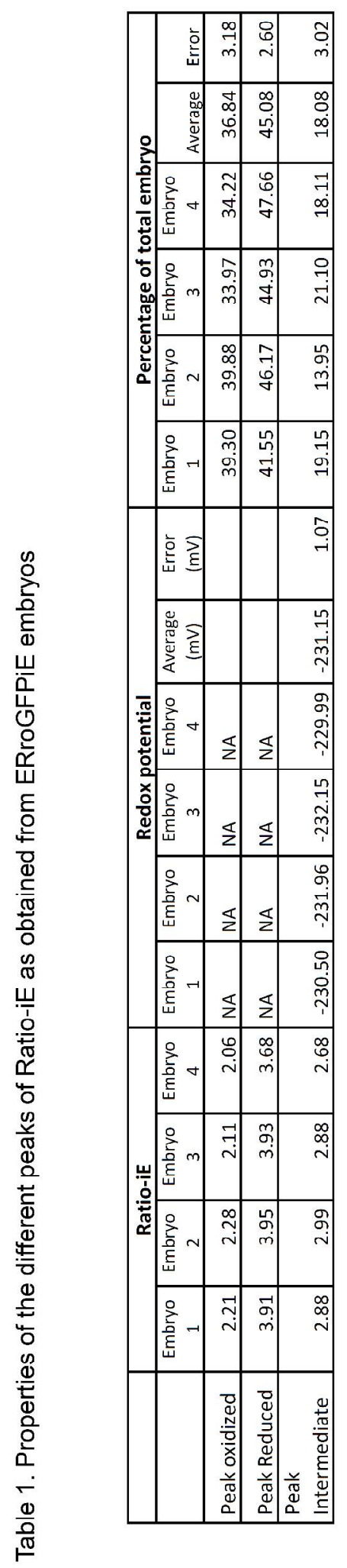




\section{Supplemental Information}

Live visualization of cytosolic and endoplasmic reticulum redox potential in zebrafish embryos reveals region-specific differences

Monika Verma, Niraj Rajesh Bhatt, Aseem Chaphalkar, Kriti Verma, Shreyansh Umale, Shweta Verma, Chetana Sachidanandan, and Kausik Chakraborty

Inventory of supplemental items

1. Figure $\mathrm{S} 1$ - relates to main Figure 1

2. Figure $S 2$ - independent

3. Figure $S 3$ - independent

4. Figure $S 4$ - relates to main Figure 3

5. Figure $S 5$ - relates to main Figure 5

6. Figure $S 6$ - relates to main Figure 6

7. Figure $S 7$ - relates to main Figure 8 
Supplementary figure 1

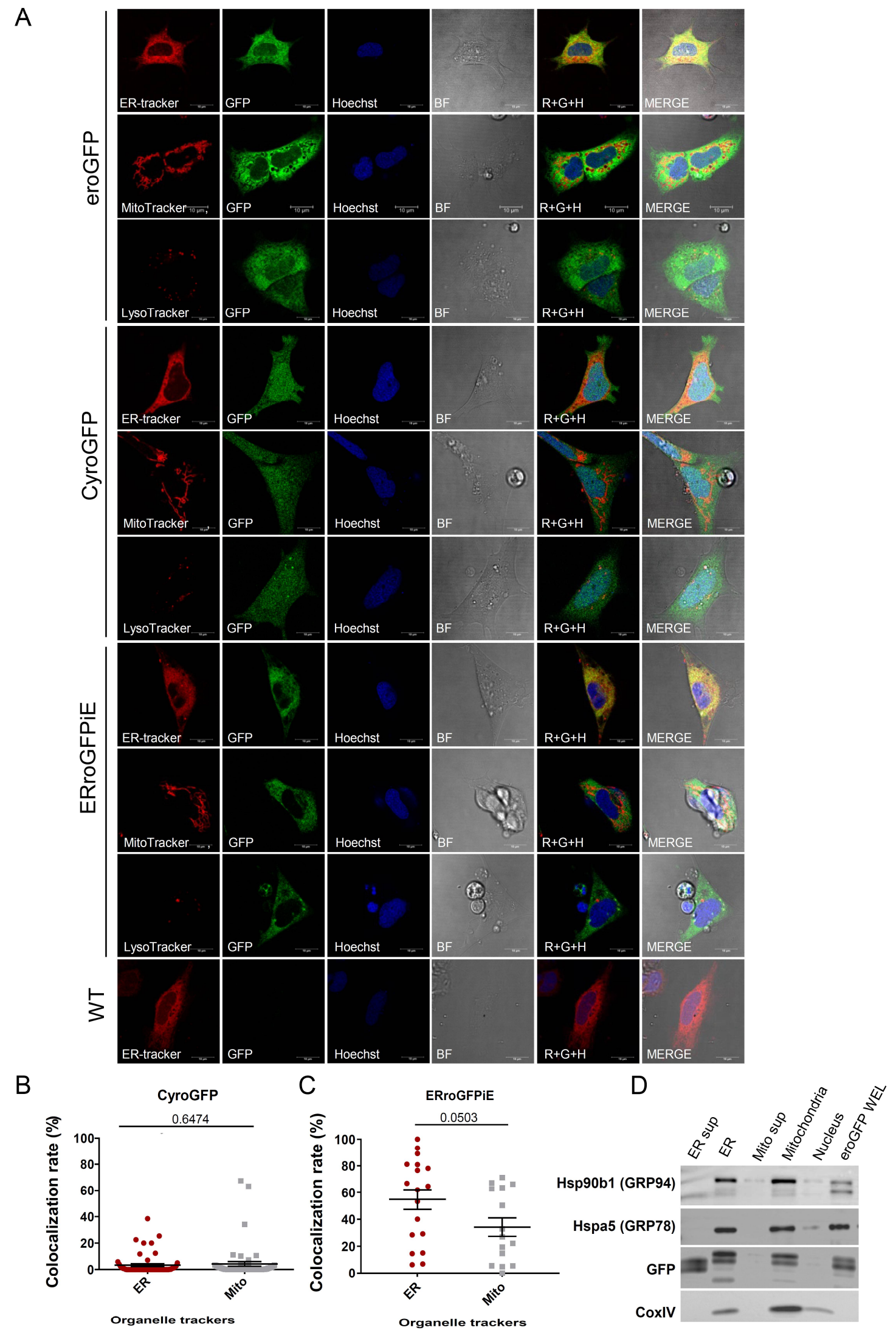


Supplementary figure 1: roGFP probes are localized in the desired organelles in generated transgenic lines. (A) Fluorescence image with ER, Mito and LysoTracker for all the transgenic lines with wild type fish as a negative control. Cytosolic line shows a diffused expression of GFP. These panels correspond to the images of transgenic lines as labeled. The first image in each panel is ER, Mito or LysoTracker followed by the GFP channel corresponding to roGFP. Hoechst was used for nuclear staining and next panel is bright field

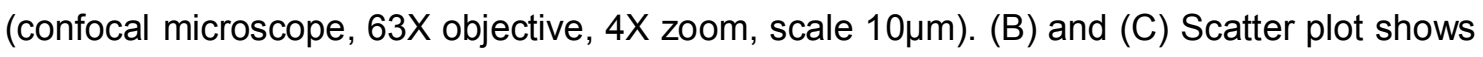
colocalization rate of green and red channel for cyroGFP and ERroGFPiE line respectively, where each dot is one ROI. (D) Western blots show fractions obtained after Subcellular fractionation which was done using sucrose and differential centrifugation. GRP78 and GRP94 are used as ER markers while COXIV as a mitochondrial marker. (BF- bright field, $\mathrm{R}+\mathrm{G}+\mathrm{H}$ - merge of red, green and hoechst channel, ER- endoplasmic reticulum, mitomitochondria, sup- supernatant, WEL- whole embryo lysate). 
Supplementary figure 2

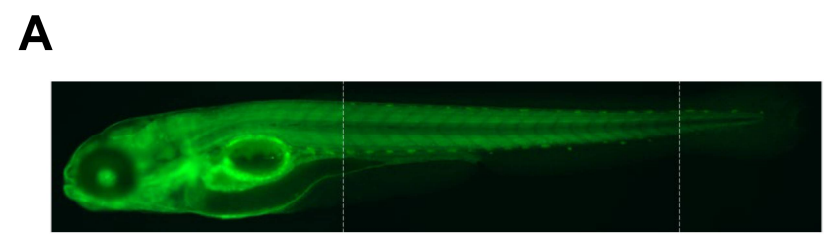

eroGFP

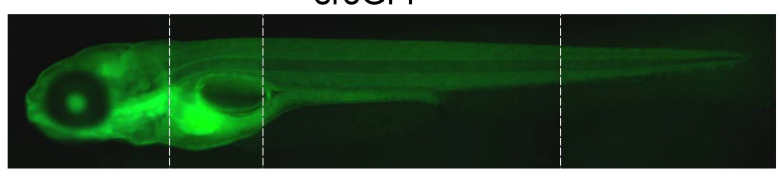

ERroGFPiE

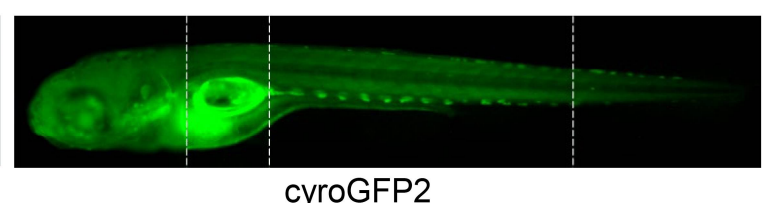

cyroGFP2

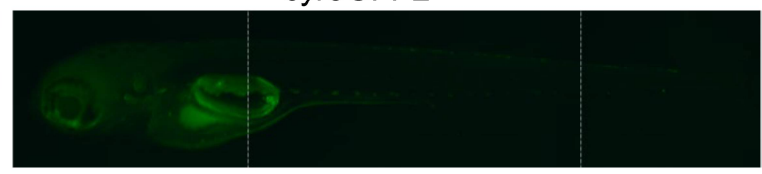

WT

Supplementary figure 2: roGFP is expressed ubiquitously in all transgenic lines. (A) Fluorescence images show $4 \mathrm{dpf}$ embryos in lateral position. Representative images of the three transgenic lines and wild type is shown (epifluorescence microscope, 5X objective). 
Supplementary figure 3

A

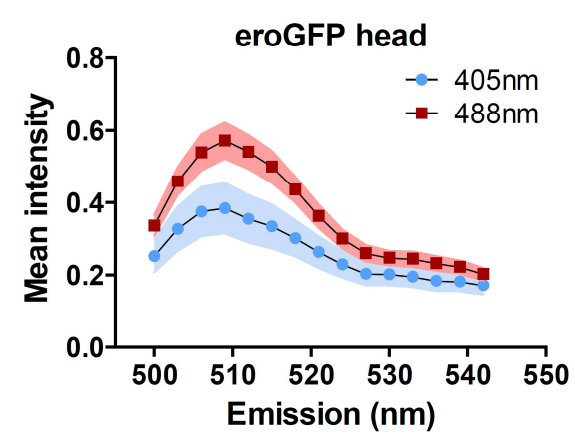

C

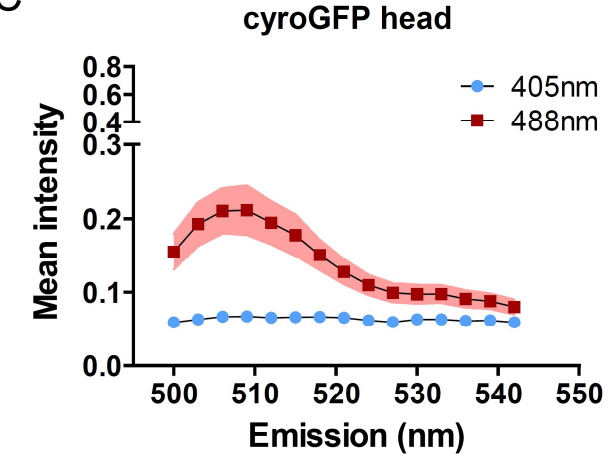

$E$

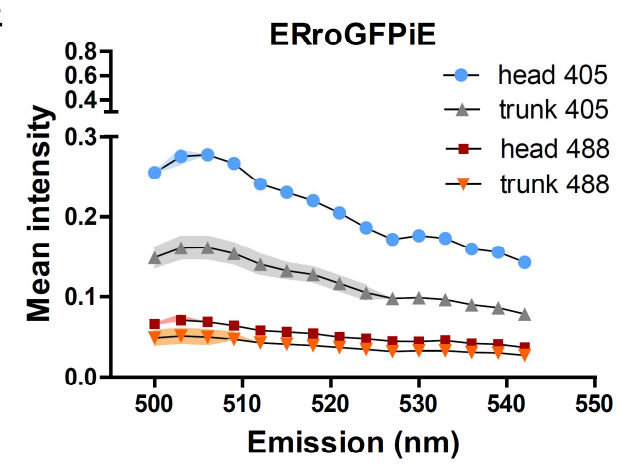

B

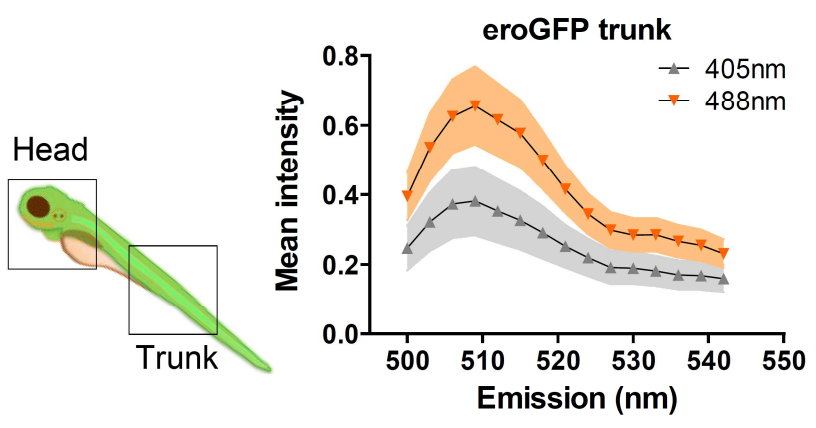

D

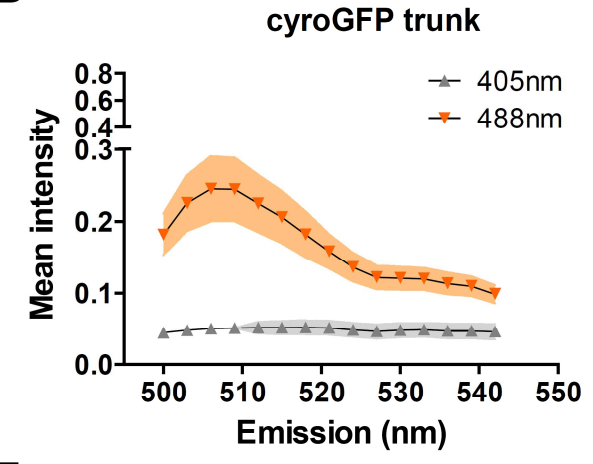

$\mathrm{F}$

Wild type

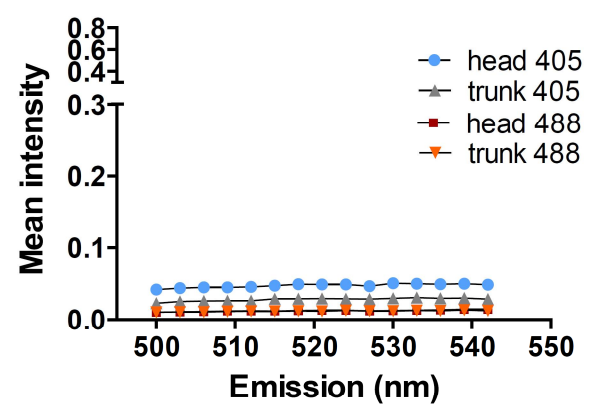

Supplementary figure 3: roGFP transgenic lines show redox sensitive properties in vivo. These graphs show emission scan for live embryos at $4 \mathrm{dpf}$ where head and trunk regions are marked in the cartoon representation of an embryo. Embryos were excited at first $405 \mathrm{~nm}$ and emission scan was taken from $500 \mathrm{~nm}$ to $540 \mathrm{~nm}$, then excited at $488 \mathrm{~nm}$ and emission was collected in the same range. (A) and (B) Graphs show emission peaks when excited at 405 and $488 \mathrm{~nm}$ for head and trunk regions of eroGFP line respectively. (C), and (D) Graphs show emission peaks at 405 and $488 \mathrm{~nm}$ for head and trunk regions of cyroGFP line respectively $(E)$ and (F) Peaks at 405 and $488 \mathrm{~nm}$ for ERroGFPiE and WT lines are shown respectively, settings were kept same for all. Each line represents mean value from 4 replicates and shaded region is SEM. (SEM- standard error around mean) 
Supplementary figure 4
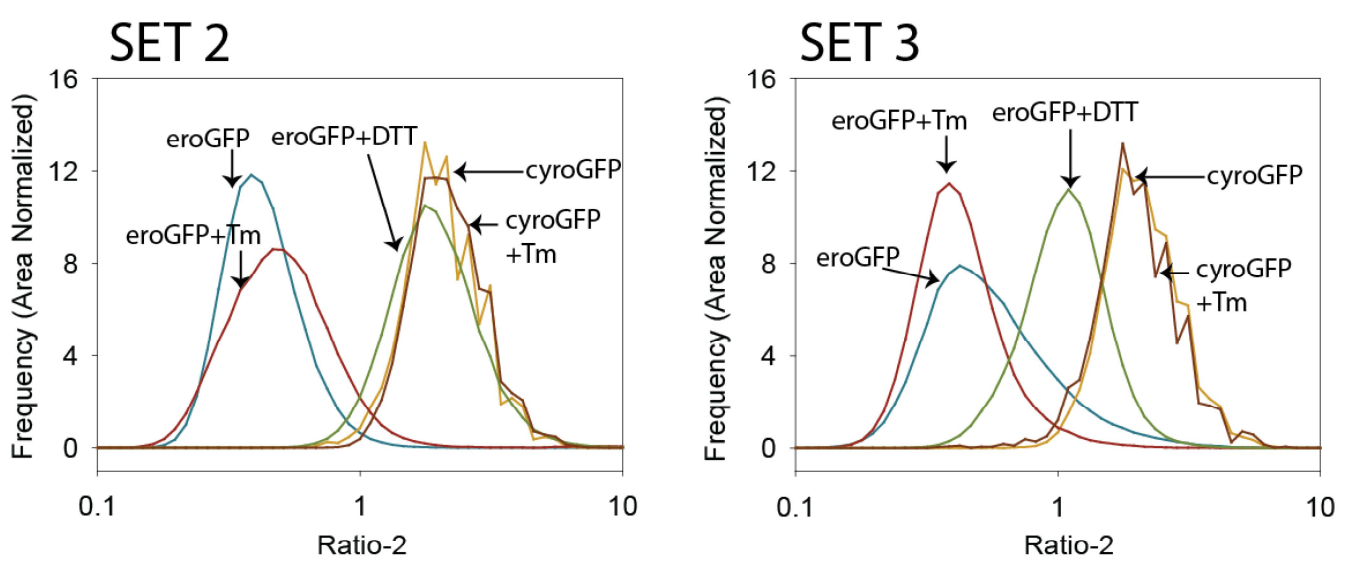

Supplementary figure 4: Ratio-2 distribution of primary cell culture from 1 dpf zebrafish embryos. Primary culture cells were treated with $6 \mu \mathrm{g} / \mathrm{ml} \mathrm{Tm}(8 \mathrm{hrs})$ and $10 \mathrm{mM}$ DTT (1-2 min). Ratio-2 values obtained after image analysis are plotted for each condition and separately for each Set. Each Set and each line represents data from one replicate and mean Ratio-2 for each treatment respectively. $\mathrm{N}$ is variable in each condition and contains value from at least 6 fields. 


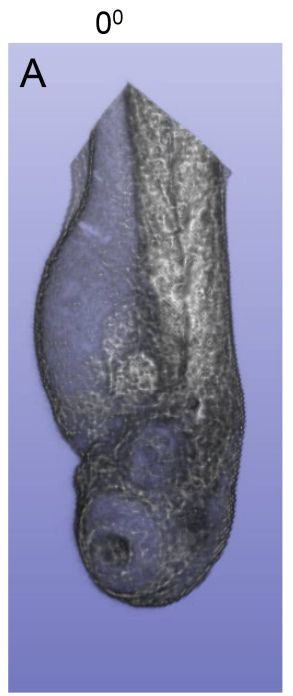

$0^{0}$

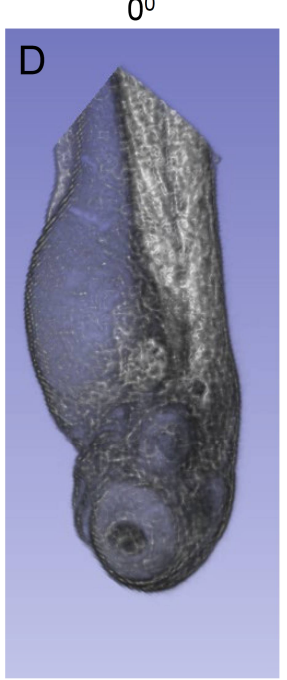

$45^{0}$

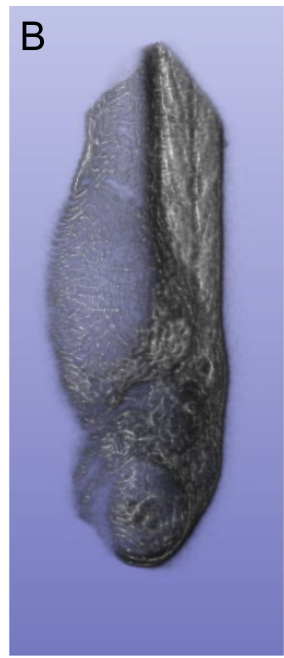

$70^{\circ}$

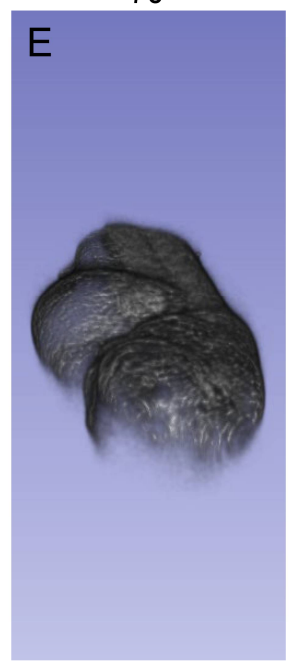

$90^{\circ}$

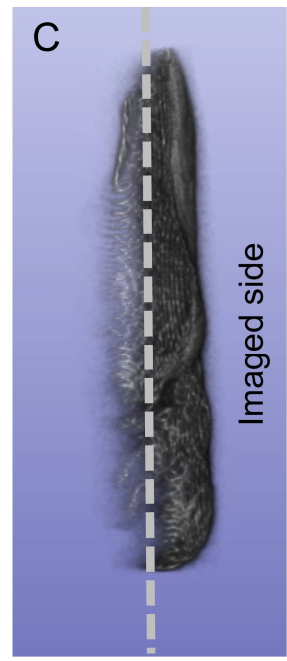

$90^{\circ}$

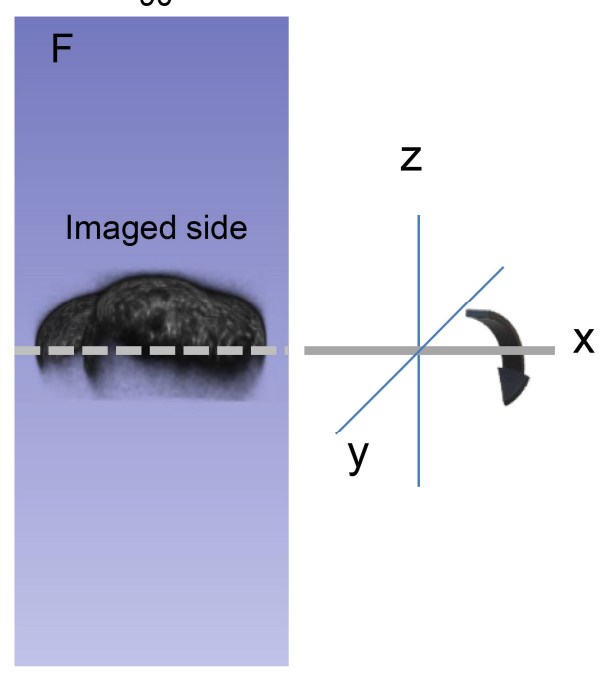

Supplementary figure 5: Confocal imaging enables to procure signals from either half of the embryo in 3D. 3D representation of a 3dpf zebrafish embryo was reconstructed from zstack images of GFP fluorescence. A single embryo is shown for illustrating the orientations used in all the figures. (A) and (D) is the $0^{\circ}$ angle of image showing the way embryo was imaged. (B) and (C) represents the embryo at $45^{\circ}$ and $90^{\circ}$ respectively when turned left to right as indicated in the arrow. $(E)$ and $(F)$ represents the embryo at $70^{\circ}$ and $90^{\circ}$ respectively when turned up from down. (Confocal microscope, 10X objective) 
Supplementary figure 6
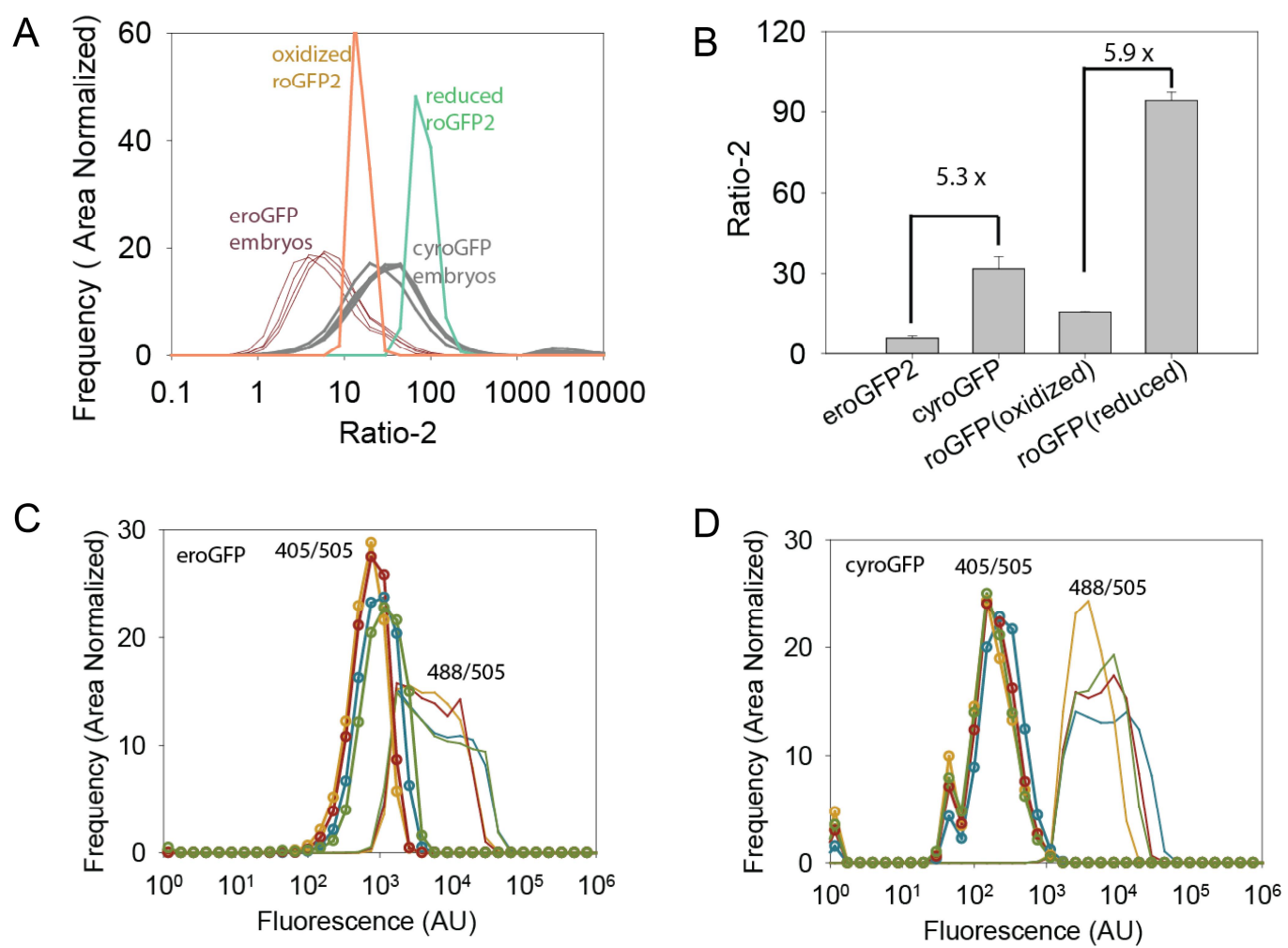

\footnotetext{
Supplementary figure 6: Ratio-2 distribution of eroGFP and cyroGFP fish lines showed shift in the peak as compared to the oxidized and reduced roGFP2 solution. $3 \mathrm{dpf}$ embryos were anaesthetized, mounted and imaged. $5 \mu \mathrm{M}$ roGFP2 solution was treated with $10 \mathrm{mM}$ DTT and $10 \mathrm{mM}$ diamide to get fully oxidized and reduced species. These solutions were also imaged using similar settings. Ratio-2 was calculated as described. (A) The frequency of each value was area normalized using the total number of pixels and graph was plotted. (B) Bar graph showing mean ratio-2 for the embryos as well as roGFP2 solution (oxidized and reduced) and error represents standard deviation. (C) and (D) Fluorescence intensities obtained with excitation at $405 \mathrm{~nm}$ and emission at 505 $\mathrm{nm}(405 / 505)$ and excitation at $488 \mathrm{~nm}$ and emission at $505 \mathrm{~nm}(488 / 505)$ were calculated for embryos. Frequency (area normalized) was plotted against fluorescence intensity for $405 / 505$ and 488/505 for eroGFP (left panel) and cyroGFP (right panel) embryos. Each line represents one embryo in (A), (B), and (D). (number of embryos, $n=4$; confocal microscope, 10X objective).
} 
Supplementary figure 7
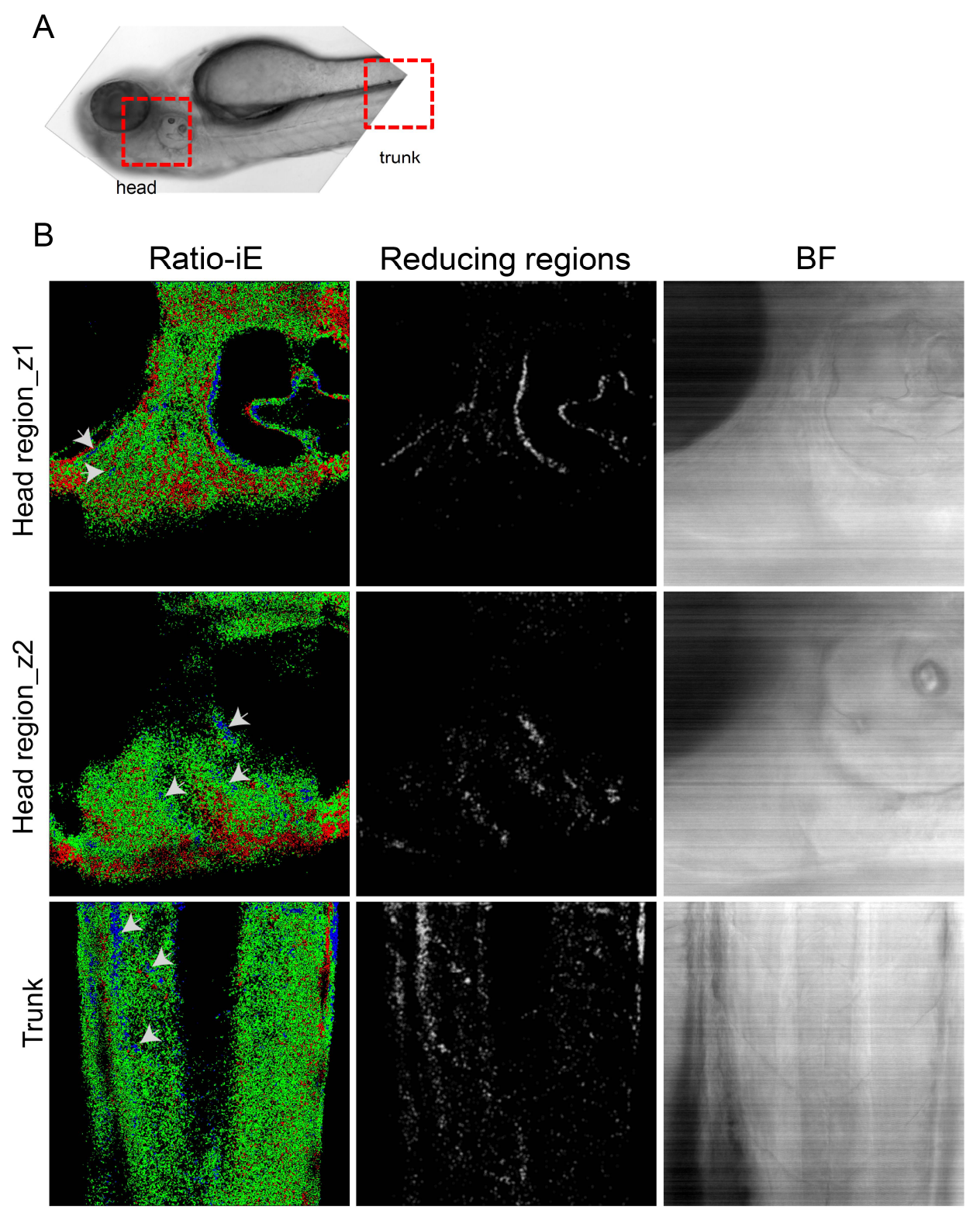

Supplementary figure 7: Variations in redox ratios of ER are more apparent with higher magnification images of ERroGFPiE line. 3 dpf live ERroGFPiE embryos were imaged, analyzed and represented similarly as earlier. (A) A 10X BF image of an embryo showing head and trunk regions by red squares imaged at higher magnification of $40 \mathrm{X}$. (B) All three panels show representative image from one embryo taken at different regions. Left, middle, and right panel show Ratio-iE, only reducing pixels, and BF images respectively. Upper panel and middle panel shows the Ratio-iE in the eye region images taken at different $z$ depths inside the embryo with some reducing regions marked with white arrows. Lower panel shows the Ratio-iE images taken at the trunk regions showing patches of reduced regions (white arrows) (BF- bright field, confocal microscope, 40X objective). 


\section{Supplementary table 1. Primers used for the generation of transgenic lines}

\begin{tabular}{|c|c|c|c|}
\hline S. no. & ame & $e\left(5^{\prime}-3^{\prime}\right)$ & otion \\
\hline 1 & ffp.infr1 & Ttttt & $\begin{array}{l}\text { fusing hspa5 ER SS with } \\
\text { roGFP }\end{array}$ \\
\hline 2 & zf.er.erogfp.fr & $\begin{array}{l}\text { Agtcagaattcatgcggttgctttgcctgtttttgctgg } \\
\text { tggccggcagcgtgtttgcc }\end{array}$ & $\begin{array}{l}\text { Amplification of zebrafish } \\
\text { eroGFP forward primer }\end{array}$ \\
\hline 3 & er.erogfp.rv & $\begin{array}{l}\text { Actac } \\
\text { attcg }\end{array}$ & \begin{tabular}{|l}
$\begin{array}{l}\text { Amplification of roGFP, } \\
\text { reverse primer }\end{array}$ \\
\end{tabular} \\
\hline 4 & zf.er.erogfp.Notl.fr & $\begin{array}{l}\text { Agtce } \\
\text { tggtg }\end{array}$ & $\begin{array}{l}\text { in of zebrafish } \\
\text { lal forward primer }\end{array}$ \\
\hline 5 & zf.er.erogfp.Clal.rv & $\begin{array}{l}\text { Acta } \\
\text { gtcc }\end{array}$ & $\begin{array}{l}\text { n of zebrafish } \\
\text { lal reverse primer }\end{array}$ \\
\hline 6 & $\begin{array}{l}\text { CyroGFP2_pSS550_for } \\
\text { ZF }\end{array}$ & $\begin{array}{l}\text { ccatc } \\
\text { cttttc }\end{array}$ & $\begin{array}{l}n \text { of } \\
\text { ner }\end{array}$ \\
\hline 7 & $\begin{array}{l}\text { CyroGFP2_pSS550_re } \\
\text { v_ZF }\end{array}$ & $\begin{array}{l}\operatorname{tctgg} \\
a \\
\end{array}$ & $\begin{array}{l}\text { ion of cyroGFP2, } \\
\text { imer }\end{array}$ \\
\hline 8 & $\begin{array}{l}\text { FiE_pSS550_i } \\
\text { ZF }\end{array}$ & $\begin{array}{l}\text { Gacs } \\
\text { gaag }\end{array}$ & $\begin{array}{l}\text { fusing hspa5 ER SS with } \\
\text { roGFP1/iE, inner forward } \\
\text { primer }\end{array}$ \\
\hline 9 & $\begin{array}{l}\text { ERroGFPiE_pSS550_f } \\
\text { or2_ZF }\end{array}$ & $\begin{array}{l}\text { Gtgg } \\
\text { agaa }\end{array}$ & $\begin{array}{l}\text { fusing hspa5 } \text { ER SS with } \\
\text { roGFP1/iE, } 2^{\text {nd }} \text { forward primer }\end{array}$ \\
\hline 10 & $\begin{array}{l}\begin{array}{l}\text { ERroGFPiE_pSS550_f } \\
\text { or3_ZF }\end{array} \\
\end{array}$ & $\begin{array}{l}\text { Atgcggttgcttgcctgttttgctggtggcc } \\
\text { cgtgtttgccga }\end{array}$ & $\begin{array}{l}\text { fusing hspa5 ER SS with } \\
\text { roGFP1/iE, } 3^{\text {rd }} \text { forward primer }\end{array}$ \\
\hline 11 & $\begin{array}{l}\text { ERroGFPiE_pSS550_o } \\
\text { ut_for4_ZF }\end{array}$ & fCalclagagchgecalgcggitge & \begin{tabular}{|lcr} 
Amplification & of & zebrafish \\
roGFP2/iE, & final & forward \\
primer & & \\
\end{tabular} \\
\hline & $\begin{array}{l}\text { ERroGFPiE_new_Rev__ } \\
\text { pSS_ZF }\end{array}$ & $\begin{array}{l}\text { Tctggatcatcatcgctaca } \\
\text { gttcatccatgc }\end{array}$ & \begin{tabular}{llr|}
$\begin{array}{l}\text { Amplification } \\
\text { of }\end{array}$ & zebrafish \\
roGFP2/iE, & final & reverse \\
primer & & \\
\end{tabular} \\
\hline
\end{tabular}

